\title{
The Static and Dynamic Transfer-Matrix Methods in the Analysis of Distributed-Feedback Lasers
}

\author{
C. A. F. Fernandes ${ }^{1}$ and José A. P. Morgado ${ }^{2}$ \\ 1,2Instituto de Telecomunicações \\ 2 Portuguese Air Force Academy \\ Portugal
}

\section{Introduction}

Numerical simulation plays a decisive role in the design of modern optical communication components. However, time efficient and user-friendly numerical techniques that perform simulations in that area are not easily available. In this chapter, an example concerning the use of a numerical simulation method, designated by transfer-matrix-method (TMM), is presented. Although the TMM is a numerical simulation tool especially adequate for the design of distributed feedback (DFB) laser structures in high bit rate optical communication systems (OCS), it represents a paradigmatic example of a numerical method related to heavy computational times.

Nowadays, distributed-feedback lasers are indispensable in high-bit rate OCS (Bornholdt et al., 2008; Sato et al., 2005; Tang et al., 2006; Utake et al., 2009; Wedding \& Pöhlmann, 2004; Wedding et al., 2003), where they should present single-longitudinal mode (SLM) operation over the largest range of current injection. In order to assure sufficient intensity modulation bandwidth in high-bit rate systems, the current injected into the laser cavity can assume high values, (Sato et al., 2005; Wedding \& Pöhlmann, 2004; Wedding et al., 2003). On the other hand, to fulfill the SLM condition, high mode selectivity and almost uniform intracavity field distributions are demanded. In the context of OCS, DFB lasers with a $90^{\circ}$ phase discontinuity near the centre of the cavity ${ }^{1}$ are commonly cited in the specialized literature, due to their high mode selectivity, small current bias and zero frequency detuning at threshold condition (Ghafouri-Shiraz, 2003). Their main drawback is related to the high non-uniformity of the field distribution along the cavity, which presents a strong peak near the centre. Above-threshold, this non-uniformity induces important variations of the carrier and refractive-index distributions arising from the spatial hole-burning (SHB) effect, with serious consequences in the laser behavior in the high power regime, namely: increased linewidth (Ghafouri-Shiraz, 2003), multi-mode emission (Morthier, 1997) and less flat laser frequency modulation response (Agrawall \& Dutta, 1986). Therefore, a careful and suitable design of the laser emitter, with a rigorous assessment of the electric field distribution along the laser cavity, is crucial in order to reduce the impact of SHB in OCS.

DFB structure analysis can be performed assuming the classical solution of the wave propagation inside periodic structures. The grating of the cavity is responsible for a

${ }^{1}$ Generally designated by quarterly wavelength-shifted (QWS) or $\lambda / 4$ DFB lasers. 
coupling between two counter-running waves, which is ruled by a pair of differential equations, usually designated by coupled-mode equations. These equations represent the essence of the theoretical analysis of longitudinal modes inside periodic laser structures, whose initial works are attributed to H. Kogelnik and C. Shank (Kogelnik \& Shank, 1972). Resonant frequencies and threshold criteria for the oscillation modes have been determined for both index and gain periodicities. However, even in the simplest case - conventional anti-reflexive (AR) coated DFB lasers - the coupled-mode equations must be solved numerically. Since the number of boundary conditions to match is generally high, the procedure will quickly become a tremendous and fastidious task for most of DFB structures. Usually, DFB structures demand the use of simulation tools more flexible than the traditional numerical techniques based on analytical or semi-analytical methods. In this scenario TMM takes place as one of the most popular and useful numerical simulation tools. It is a method that can easily handle with complex periodic structures, both for static and dynamic regimes. The same generic algorithm may be used in a straightforward way for the analysis of any kind of multi-section laser structures, namely, multiple phase-shift (MPS)DFB (Tan et al., 1995), distributed-coupled coefficient (DCC)-DFB (Ghafouri-Shiraz, 2003 ; Boavida et al., 2011), corrugation pitch modulated (CPM)-DFB (Fessant, 1997), multielectrode DFB (Ghafouri-Shiraz, 2003), distributed Bragg reflector (DBR) (Lowery, 1991), vertical cavity (Yu, 2003), etc, as long as the perturbation included in the periodic chain may be described by a transfer matrix. A detailed description of those numerical techniques will be the scope of this work. However, as previously referred, matrix methods are usually very heavy in terms of processing times and so they should be optimized in order to improve their time computational efficiency. The search for adequate strategies aiming at an efficient convergence of the TMM, both for static and dynamic regimes, is crucial and it will be one of the leit-motiv of the present study. Accordingly, a convenient approach to TMM suggests an introduction to the coupled wave theory.

\section{The coupled wave theory}

In a homogeneous, source-free and lossless medium, any time harmonic electric field obeys the vector wave equation

$$
\nabla^{2} \bar{E}+k_{0}^{2} \cdot n^{2} \cdot \bar{E}=0
$$

In (1) the time dependence, $t$, of the electric field has been assumed to be $e^{j \omega t}$, with $\omega$ the angular frequency, $\bar{E}$ the complex amplitude of the electric field, $n$ the refractive index and $k_{0}$ the free space propagation constant. From Maxwell equations it is possible to show that in a semiconductor laser, which has an oscillating lateral and transversal confined electric field, the longitudinal wave propagation obeys the one-dimensional homogeneous wave equation

$$
\frac{d^{2} \bar{E}(z)}{d z^{2}}+\bar{k}^{2}(z) \cdot \bar{E}(z)=0,
$$

commonly referred as the scalar wave-equation. In $(2) \bar{k}(z)$ is the complex propagation constant related to $\bar{E}$, given by

$$
\bar{k}(z)=\beta(z)+j g_{u}(z),
$$


where $\beta(z)$ is the phase propagation constant by unit length and $g_{u}(z)$ is the amplitude gain by unit length associated with the propagation of the electric field along the cavity. In DFB lasers the corrugation-induced dielectric perturbation along the laser cavity (grating) leads to a periodic Bragg waveguide and, therefore, to the longitudinal dependence of the propagation constants. These are given by

$$
\beta(z) \triangleq \frac{2 \pi f}{c_{\Lambda}(z)}
$$

where $f$ is the frequency and $c_{\Lambda}(z)$ is the propagation velocity of $\bar{E}$ inside the Bragg waveguide, given by

$$
c_{\Lambda}(z) \triangleq \frac{1}{\sqrt{\mu(z) \cdot \varepsilon(z)}} .
$$

In (5) $\mu$ represents the magnetic permeability, usually given for non-magnetic materials by its value in free space $\mu_{0}=4 \pi \times 10^{-7} \mathrm{H} \cdot \mathrm{m}^{-1}$, and $\varepsilon$ is material permittivity $\left(\varepsilon_{0}=8.854 \times 10^{-12} \mathrm{~F} \cdot \mathrm{m}^{-1}\right.$ for free space). Substituting (5) in (4), it yields

$$
\beta(z)=2 \pi f \cdot \sqrt{\varepsilon(z) \cdot \mu(z)} \cong 2 \pi f \cdot \sqrt{\varepsilon_{0} \cdot \mu_{0}} \frac{\sqrt{\varepsilon(z) \cdot \mu_{0}}}{\sqrt{\varepsilon_{0} \cdot \mu_{0}}} \cong k_{0} \cdot n(z),
$$

with the free-space phase propagation constant and the semiconductor refractive index given, respectively, by

$$
k_{0} \triangleq 2 \pi f \cdot \sqrt{\mu_{0} \cdot \varepsilon_{0}} ; n(z) \cong \frac{\sqrt{\mu_{0} \cdot \varepsilon(z)}}{\sqrt{\mu_{0} \cdot \varepsilon_{0}}}=\sqrt{\frac{\varepsilon(z)}{\varepsilon_{0}}} .
$$

Assuming that the corrugation has a period $\Lambda$, it is implicitly assumed that the refractive index and the amplitude gain are also periodic functions of the same period. Since the length of the laser cavity (commonly hundreds of micrometers) is much longer than $\Lambda$ (generally, some nanometers), it is possible to represent the waveguide by a Fourier series. In this approach, the Bragg waveguide may be considered a first-order waveguide, leading to

$$
n(z) \cong n_{0}+\Delta n \cdot \cos \left(2 \pi \cdot \frac{z}{\Lambda}+\Omega_{\Lambda}\right) ; g_{u}(z) \cong g_{u 0}+\Delta g_{u} \cdot \cos \left(2 \pi \cdot \frac{z}{\Lambda}+\Omega_{\Lambda}+\theta_{\Lambda}\right) .
$$

In (8), where only the first two terms of the series have been considered, $n_{0}$ and $g_{u 0}$ are the mean values of $n(z)$ and $g_{u}(z)$, respectively, and $\Delta n$ and $\Delta g_{u}$ are their modulation amplitudes. $\Omega_{\Lambda}$ is the phase of the periodic variations of the refractive index for $z=0$ and $\theta_{\Lambda}$ is the relative phase deviation between the perturbations $n(z)$ and $g_{u}(z)$. Hereafter, $g_{u 0}$ will be designated by $\alpha$. The period $\Lambda$ imposes some restrictions to the values that $\beta(z)$ can assume. In Fig. 1 it is schematically shown the propagation of the electric field in a periodic structure, assuming $g_{u}(z)=1 \mathrm{~m}^{-1}$. 


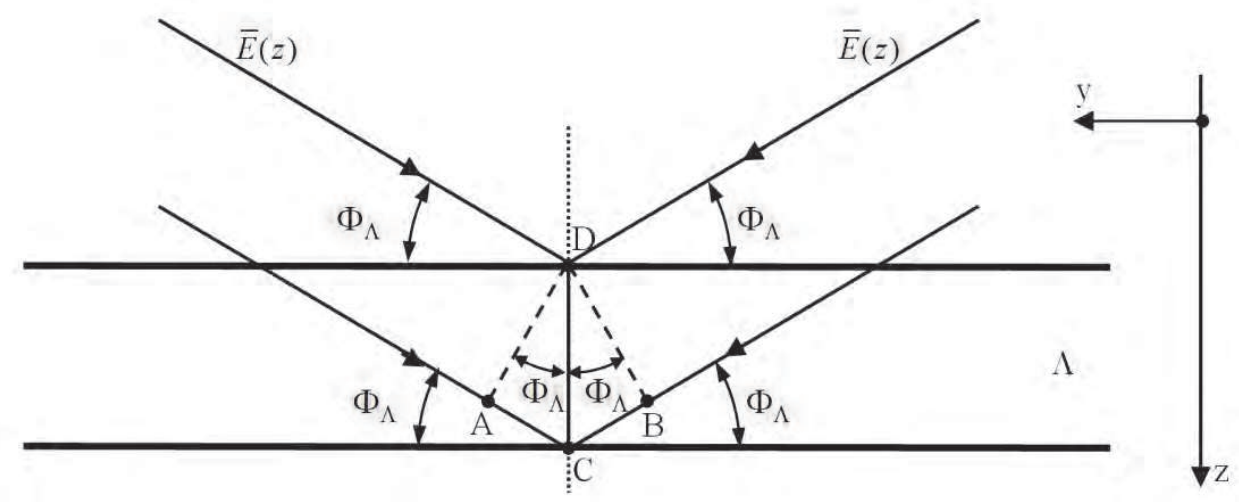

Fig. 1. Electric field propagation in a periodic waveguide, assuming lateral and transversal confinements of $\bar{E}(z)$.

For $N_{\Lambda}$ periods, $N_{\Lambda}$ reflected waves will be generated. Their positive interference demands that the phase difference between two reflected waves be a multiple of $2 \pi$. According to Fig. 1 this means that

$$
\beta(z) \cdot(\overline{A B}+\overline{B C})=\beta(z) \cdot\left[2 \Lambda \cdot \sin \left(\Phi_{\Lambda}\right)\right]=2 m \pi \quad: \quad m=1 ; 2 ; 3 \ldots .
$$

Assuming $\Phi_{\Lambda}=90^{\circ}$, for a first order corrugation it yields $\beta(z)=\pi / \Lambda$. Therefore, the propagation constant is independent of $z$, being known as the Bragg propagation constant $\beta_{\Lambda}=\pi / \Lambda$. Physically, it represents a value in the vicinity of which the propagation constant must be included so that the electric field may propagate along the structure. Related to this value, it may be defined the structure wavelength $\lambda_{\text {struct }} \triangleq 2 \pi / \beta_{\Lambda}=2 \Lambda$. This parameter may be related to an oscillator whose frequency is designated by the Bragg frequency, which is given by

$$
f_{\Lambda} \triangleq \frac{c}{\lambda_{\text {struct }} \cdot n_{0}}
$$

In (10) $c$ is the free-space velocity. For an oscillator in free-space of the same frequency, the wavelength is known as the Bragg wavelength, and it is given by

$$
\lambda_{\Lambda} \triangleq \frac{c}{f_{\Lambda}}=\lambda_{\text {struct }} \cdot n_{0}=2 \Lambda \cdot n_{0}
$$

Assuming that $g_{u}(z) \ll \beta(z), \Delta n \ll n_{0}$ and $\Delta g_{u} \ll \alpha$, it is possible, after some algebraic manipulation, to show that

$$
\begin{aligned}
& \bar{k}^{2}(z) \cong \beta^{2}(z)+j 2 \cdot \beta(z) \cdot \alpha+2 \cdot \beta(z) \cdot \bar{\kappa}_{R \leftarrow S} \cdot \exp \left[-j\left(2 \cdot \beta_{\Lambda} \cdot z+\Omega_{\Lambda}\right)\right]+ \\
& +2 \cdot \beta(z) \cdot \bar{\kappa}_{S \leftarrow R} \cdot \exp \left[j\left(2 \cdot \beta_{\Lambda} \cdot z+\Omega_{\Lambda}\right)\right]
\end{aligned}
$$

with $\bar{\kappa}_{R \leftarrow S}$ and $\bar{\kappa}_{S \leftarrow R}$ being the coupling coefficients related to the Bragg waveguide, which are given by 


$$
\bar{\kappa}_{R \leftarrow S} \triangleq \frac{\pi \cdot \Delta n}{\lambda_{0}}+j \cdot \frac{\Delta g_{u}}{2} \cdot \exp \left(-j \cdot \theta_{\Lambda}\right) ; \bar{\kappa}_{S \leftarrow R} \triangleq \frac{\pi \cdot \Delta n}{\lambda_{0}}+j \cdot \frac{\Delta g_{u}}{2} \cdot \exp \left(j \cdot \theta_{\Lambda}\right)
$$

with

$$
\lambda_{0} \triangleq \frac{2 \pi}{k_{0}}
$$

Considering $\kappa_{R} \triangleq \pi \cdot \Delta n / \lambda_{0}$ and $\kappa_{I} \triangleq \Delta g_{u} / 2$, it is possible to write the coupling coefficients (13) as

$$
\bar{\kappa}_{R \leftarrow S}=\kappa_{R}+j \cdot \kappa_{I} \cdot \exp \left(-j \cdot \theta_{\Lambda}\right) ; \bar{\kappa}_{S \leftarrow R}=\kappa_{R}+j \cdot \kappa_{I} \cdot \exp \left(j \cdot \theta_{\Lambda}\right) .
$$

From (15), it may be seen that the contributions for the coupling coefficients, related to the perturbations in the refractive index or in the gain per unit length, are included in $\kappa_{R}$ and $\kappa_{I}$, respectively. Using (12) in (2), it yields

$$
\begin{aligned}
\frac{d^{2} \bar{E}(z)}{d z^{2}}+\beta^{2}(z) \cdot \bar{E}(z)+j \cdot 2 \beta(z) \cdot \alpha \cdot \bar{E}(z) & +2 \beta(z) \cdot\left\{\bar{\kappa}_{R \leftarrow S} \cdot \exp \left[-j\left(2 \beta_{\Lambda} \cdot z+\Omega_{\Lambda}\right)\right]\right\} \cdot \bar{E}(z)+ \\
& +2 \beta(z) \cdot\left\{\bar{\kappa}_{S \leftarrow R} \cdot \exp \left[j\left(2 \beta_{\Lambda} \cdot z+\Omega_{\Lambda}\right)\right]\right\} \cdot \bar{E}(z)=0 .
\end{aligned}
$$

Equation (16) gives the electric field profile considering the global effects of the lateral and transversal confinements and the presence of a corrugation in the laser cavity.

Without corrugation, which is the case of the Fabry-Pérot (FP) lasers, $\bar{\kappa}_{R \leftarrow S}=\bar{\kappa}_{S \leftarrow R}=\Delta n=\Delta g_{u}=0$. Therefore, from (16), results

$$
\frac{d^{2} \bar{E}(z)}{d z^{2}}+\left[\beta^{2}+j \cdot 2 \beta \cdot \alpha\right] \cdot \bar{E}(z)=0 .
$$

Taking into account that in this case $g_{u}(z)=\alpha \ll \beta(z)=\beta$, it yields

$$
\beta^{2}+j \cdot 2 \beta \cdot \alpha \cong \beta^{2}+j \cdot 2 \beta \cdot \alpha+\alpha^{2}=(\beta+j \alpha)^{2}
$$

Being (17) a linear homogeneous ordinary differential equation with constant coefficients, the solution may be written as

$$
\bar{E}(z)=\bar{A}(z) \cdot \exp (-j \cdot \beta \cdot z)+\bar{B}(z) \cdot \exp (j \cdot \beta \cdot z),
$$

where $\bar{A}(z)$ and $\bar{B}(z)$ are arbitrary complex constants to be determined according to the boundary conditions imposed at the cavity ends.

Let us now assume a DFB laser, that is, a laser with a corrugation inside the cavity, which is responsible for an amount of feedback distributed along the cavity. The solution is seen to be formally identical to the one obtained in (19) but with $\beta$ replaced by $\beta(z)$, which is in the vicinity of the Bragg propagation constant so that positive interference should happen. Therefore, it may be assumed that

$$
\left|\beta(z)-\beta_{\Lambda}\right| \ll \beta_{\Lambda}
$$


with the detuning defined as

$$
\delta \triangleq \beta(z)-\beta_{\Lambda}
$$

According to (20) and (21), (19) may be rewritten as

$$
\bar{E}(z)=\bar{R}(z) \cdot \exp \left(-j \cdot \beta_{\Lambda} \cdot z\right)+\bar{S}(z) \cdot \exp \left(j \cdot \beta_{\Lambda} \cdot z\right),
$$

where

$$
\bar{R}(z) \triangleq \bar{A}(z) \cdot \exp (-j \cdot \delta \cdot z) ; \bar{S}(z) \triangleq \bar{B}(z) \cdot \exp (j \cdot \delta \cdot z)
$$

Equation (22) shows that $\bar{E}(z)$ is the superposition of two counter-running waves, $\bar{R}(z)$ and $\bar{S}(z)$. Using (22) in (16), it is obtained after some algebraic manipulation

$$
\begin{aligned}
& -\frac{d \bar{R}(z)}{d z}+(\alpha-j \delta) \cdot \bar{R}(z)=j \bar{\kappa}_{R \leftarrow S} \bar{S}(z) \cdot \exp \left(-j \cdot \Omega_{\Lambda}\right) ; \\
& \frac{d \bar{S}(z)}{d z}+(\alpha-j \delta) \cdot \bar{S}(z)=j \bar{\kappa}_{S \leftarrow R} \bar{R}(z) \cdot \exp \left(j \cdot \Omega_{\Lambda}\right) .
\end{aligned}
$$

Equations (24) are known as the coupled-wave equations. It should be emphasized that (24) is valid for periodic laser cavities as far as the included perturbations are weak. The physical meaning of the coupling coefficients $\bar{\kappa}_{R \leftarrow S}$ and $\bar{\kappa}_{S \leftarrow R}$ is clearly described by (24). They represent the amount of feedback per unit length in the propagation along the cavity. This means that there is a net energy transfer between the two counter-running waves associated to the electric field distribution. The coupling coefficient $\bar{\kappa}_{R \leftarrow S}$ measures the coupling that $\bar{S}(z)$ induces in $\bar{R}(z)$. It is known as the forward coupling coefficient. The coupling coefficient $\bar{\kappa}_{S \leftarrow R}$ measures the coupling that $\bar{R}(z)$ induces in $\bar{S}(z)$. It is known as the backward coupling coefficient.

In the following, some applications of the coupled-wave theory in the analysis of the threshold regime related to simple laser structures will be considered.

\subsection{Threshold analysis of conventional DFB lasers with reflective facets}

Let us consider a conventional DFB laser structure, which is a DFB laser with a constant period grating defined by $\Lambda(z)=\Lambda$. The cavity ends (facets) will be defined by their reflectivities, $\hat{r}_{1}$ and $\hat{r}_{2}$, at left and right facets, respectively. Without loss of generality, it will be considered hereafter $\theta_{\Lambda}=0$. From (15) and (8), it yields

$$
\begin{aligned}
& \bar{\kappa}_{R \leftarrow S}=\bar{\kappa}_{S \leftarrow R}=\kappa_{R}+j \cdot \kappa_{I} ; \\
& n(z) \cong n_{0}+\Delta n \cdot \cos \left[\Psi_{\Lambda}(z)\right] ; \\
& g_{u}(z) \cong \alpha+\Delta g_{u} \cdot \cos \left[\Psi_{\Lambda}(z)\right],
\end{aligned}
$$

where $\Psi_{\Lambda}(z) \triangleq 2 \beta_{\Lambda} \cdot z+\Omega_{\Lambda}$ represents the corrugation phase at $z$ coordinate.

Since the forward and backward coupling coefficients are equal, they will be referred as the coupling coefficient and represented by $\bar{\kappa}$. According to (24) the solution of the coupledwave equations may be written as 


$$
\begin{aligned}
& \bar{R}(z)=\bar{R}_{1}(z) \cdot \exp (\bar{\gamma} \cdot z)+\bar{R}_{2}(z) \cdot \exp (-\bar{\gamma} \cdot z) \\
& \bar{S}(z)=\bar{S}_{1}(z) \cdot \exp (\bar{\gamma} \cdot z)+\bar{S}_{2}(z) \cdot \exp (-\bar{\gamma} \cdot z) .
\end{aligned}
$$

From (22) and (26), it yields

$$
\begin{aligned}
& \bar{E}(z)=\left[\bar{R}_{1}(z) \cdot \exp (\bar{\gamma} \cdot z)+\bar{R}_{2}(z) \cdot \exp (-\bar{\gamma} \cdot z)\right] \cdot \exp \left(-j \beta_{\Lambda} \cdot z\right)+ \\
& +\left[\bar{S}_{1}(z) \cdot \exp (\bar{\gamma} \cdot z)+\bar{S}_{2}(z) \cdot \exp (-\bar{\gamma} \cdot z)\right] \cdot \exp \left(j \beta_{\Lambda} \cdot z\right) .
\end{aligned}
$$

In the previous equations, $\bar{R}_{1}(z), \bar{R}_{2}(z), \bar{S}_{1}(z), \bar{S}_{2}(z)$ and $\bar{\gamma}(z)$ are complex values that are determined according to the boundary conditions imposed at the cavity facets. However, no matter the type of facets considered, it is possible to show that, in order that the solution (27) is non-trivial, the following condition should always be verified

$$
\bar{\gamma}^{2}=(\alpha-j \delta)^{2}+\bar{\kappa}^{2}
$$

This condition is generally known as the dispersion equation. For a cavity with length $L$ and reflecting facets with reflectivities $\hat{r}_{1}$ and $\hat{r}_{2}$, respectively, at left and right ends, the boundary conditions at the cavity ends impose that

$$
\begin{aligned}
& \bar{R}\left(z_{1}\right) \cdot \exp \left(-j \bar{\beta}_{\Lambda} \cdot z_{1}\right)=\widehat{r}_{1} \cdot \bar{S}\left(z_{1}\right) \cdot \exp \left(j \bar{\beta}_{\Lambda} \cdot z_{1}\right) \\
& \bar{S}\left(z_{2}\right) \cdot \exp \left(j \bar{\beta}_{\Lambda} \cdot z_{2}\right)=\widehat{r}_{2} \cdot \bar{R}\left(z_{2}\right) \cdot \exp \left(-j \bar{\beta}_{\Lambda} \cdot z_{2}\right) .
\end{aligned}
$$

From (29) and the dispersion equation (28) it is obtained, after some algebraic manipulations

$$
\bar{\gamma} L=\frac{-j \bar{\kappa} \cdot L \cdot \sinh (\bar{\gamma} L)}{D} \cdot\left[\left(\hat{r}_{1}+\hat{r}_{2}\right) \cdot\left(1-\hat{r}_{1} \cdot \hat{r}_{2}\right) \cdot \cosh (\bar{\gamma} L) \pm\left(1+\hat{r}_{1} \cdot \hat{r}_{2}\right) \cdot \varsigma^{0.5}\right]
$$

with

$$
\begin{aligned}
& \varsigma \triangleq\left(\hat{r}_{1}-\hat{r}_{2}\right)^{2} \cdot \sinh ^{2}(\bar{\gamma} L)+\left(1-\hat{r}_{1} \cdot \hat{r}_{2}\right)^{2} \\
& D \triangleq\left(1+\hat{r}_{1} \cdot \hat{r}_{2}\right)^{2}-4 \cdot \hat{r}_{1} \cdot \hat{r}_{2} \cdot \cosh ^{2}(\bar{\gamma} L) \\
& \hat{r}_{1}=r_{1} \cdot \exp \left[j\left(2 \beta_{\Lambda} \cdot z_{1}+\Omega_{\Lambda}\right)\right]=r_{1} \cdot \exp \left(\Psi_{\Lambda_{1}}\right) \\
& \hat{r}_{2}=r_{2} \cdot \exp \left[-j\left(2 \beta_{\Lambda} \cdot z_{2}+\Omega_{\Lambda}\right)\right]=r_{2} \cdot \exp \left(\Psi_{\Lambda_{2}}\right) .
\end{aligned}
$$

In (31) $\Psi_{\Lambda_{1}}$ is the corrugation phase at the left facet $\left(z=z_{1}\right)$ and $\Psi_{\Lambda_{2}}$ is the symmetric of the corrugation phase at the right facet $\left(z=z_{2}\right)$. Equation (30) represents the threshold condition for the conventional DFB laser. It depends on the coupling coefficient, the cavity length and the reflectivities at both cavity ends. Its solution allows the determination of the detuning, $\delta$, and the gains associated with all modes, $\alpha$, that are allowed to propagate inside the cavity. For most practical cases it recurs to numerical methods, taking into account that it may be rewritten in a more suitable form as 


$$
\begin{aligned}
& (\bar{\gamma} L)^{2} \cdot D+(\bar{\kappa} \cdot L)^{2} \cdot \sinh ^{2}(\bar{\gamma} L) \cdot\left(1-\hat{r}_{1}^{2}\right) \cdot\left(1-\hat{r}_{2}^{2}\right)+ \\
& +j 2(\bar{\kappa} \cdot L) \cdot\left(\hat{r}_{1}+\hat{r}_{2}\right) \cdot\left(1-\hat{r}_{1} \cdot \hat{r}_{2}\right) \cdot(\bar{\gamma} L) \cdot \sinh (\bar{\gamma} L) \cdot \cosh (\bar{\gamma} L)=0 .
\end{aligned}
$$

The solutions of (32) are seen to depend strongly on the conditions at the cavity ends, through the values assumed for their reflectivities, both in amplitude and phase. This may be problematic as far as the laser characterization is concerned, since due to fabrication limitations the values assumed for the phases in the facet reflectivities are known with a certain degree of uncertainty.

To solve the complex equation (32), Newton-Raphson iteration techniques are generally used, provided the Cauchy-Riemann condition on complex analytical functions are satisfied. In the following paragraphs it is briefly presented a generalization of the usual NewtonRaphson method for the solution of non-linear real equations. Let us then consider the following generic equation of an arbitrary complex function of a complex variable,

$$
\bar{W}(\bar{z})=0,
$$

and let us assume that $\bar{W}(\bar{z})$ is an analytical function, i.e., that it is possible to calculate $d \bar{W}(\bar{z}) / d \bar{z}$ in the vicinity of $\bar{z}$. The complex function is described as

$$
\bar{W}(\bar{z})=U(\bar{z})+j V(\bar{z}) .
$$

In (34) $U(\bar{z})$ and $V(\bar{z})$ are real functions that represent the real and imaginary parts of $\bar{W}(\bar{z})$, respectively. Taking into account that $\bar{z}=x+j y$, the solution of (34) is equivalent to the solution of the following system of equations

$$
U(x, y)=0 ; V(x, y)=0 .
$$

The Taylor expansion of (35) in the vicinity of an approximate solution $\left(x_{0}, y_{0}\right)$ yields to

$$
\begin{gathered}
U\left(x_{1}, y_{1}\right)=U\left(x_{0}, y_{0}\right)+\left.\frac{\partial U(x, y)}{\partial x}\right|_{x_{0}, y_{0}} \cdot\left(x_{1}-x_{0}\right)+\left.\frac{\partial U(x, y)}{\partial y}\right|_{x_{0}, y_{0}} \cdot\left(y_{1}-y_{0}\right) \\
V\left(x_{1}, y_{1}\right)=V\left(x_{0}, y_{0}\right)+\left.\frac{\partial V(x, y)}{\partial x}\right|_{x_{0}, y_{0}} \cdot\left(x_{1}-x_{0}\right)+\left.\frac{\partial V(x, y)}{\partial y}\right|_{x_{0}, y_{0}} \cdot\left(y_{1}-y_{0}\right) .
\end{gathered}
$$

From (35), (36) and (37), it yields

$$
U\left(x_{1}, y_{1}\right) \cong 0 ; \quad V\left(x_{1}, y_{1}\right) \cong 0 ，
$$

where $x_{1}$ and $y_{1}$ are in the vicinity of $x_{0}$ and $y_{0}$. Using (38) in (36) and (37), it is obtained

$$
\left.\frac{\partial U(x, y)}{\partial x}\right|_{x_{0}, y_{0}} \cdot x_{1}+\left.\frac{\partial U(x, y)}{\partial y}\right|_{x_{0}, y_{0}} \cdot y_{1}=\left[-U\left(x_{0}, y_{0}\right)+\left.\frac{\partial U(x, y)}{\partial x}\right|_{x_{0}, y_{0}} \cdot x_{0}+\left.\frac{\partial U(x, y)}{\partial y}\right|_{x_{0}, y_{0}} \cdot y_{0}\right]
$$




$$
\left.\frac{\partial V(x, y)}{\partial x}\right|_{x_{0}, y_{0}} \cdot x_{1}+\left.\frac{\partial V(x, y)}{\partial y}\right|_{x_{0}, y_{0}} \cdot y_{1}=\left[-V\left(x_{0}, y_{0}\right)+\left.\frac{\partial V(x, y)}{\partial x}\right|_{x_{0}, y_{0}} \cdot x_{0}+\left.\frac{\partial V(x, y)}{\partial y}\right|_{x_{0}, y_{0}} \cdot y_{0}\right] \text {. }
$$

The solutions $x_{1}$ and $y_{1}$ of the previous system of equations are

$$
\begin{aligned}
& x_{1}=x_{0}+\frac{\left.V\left(x_{0}, y_{0}\right) \cdot \frac{\partial U(x, y)}{\partial y}\right|_{x_{0}, y_{0}}-\left.U\left(x_{0}, y_{0}\right) \cdot \frac{\partial V(x, y)}{\partial y}\right|_{x_{0}, y_{0}}}{\Delta_{\text {Newt }}} \\
& y_{1}=y_{0}+\frac{\left.U\left(x_{0}, y_{0}\right) \cdot \frac{\partial V(x, y)}{\partial x}\right|_{x_{0}, y_{0}}-\left.V\left(x_{0}, y_{0}\right) \cdot \frac{\partial U(x, y)}{\partial x}\right|_{x_{0}, y_{0}}}{\Delta_{\text {Newt }}},
\end{aligned}
$$

where

$$
\Delta_{\text {Newt }}=\left.\left.\frac{\partial U(x, y)}{\partial x}\right|_{x_{0}, y_{0}} \cdot \frac{\partial V(x, y)}{\partial y}\right|_{x_{0}, y_{0}}-\left.\left.\frac{\partial V(x, y)}{\partial x}\right|_{x_{0}, y_{0}} \cdot \frac{\partial U(x, y)}{\partial y}\right|_{x_{0}, y_{0}}
$$

Taking into account (34), it is straightforward that ${ }^{2} \quad U(x, y)=\Re[\bar{W}(\bar{z})]$ and $V(x, y)=\mathfrak{I}[\bar{W}(\bar{z})]$, which yields

$$
\frac{d \bar{W}(\bar{z})}{d \bar{z}}=\frac{d U(\bar{z})}{d \bar{z}}+j \frac{d V(\bar{z})}{d \bar{z}} .
$$

From $\bar{z}=x+j y$, and (43) one obtains the following equations

$$
\begin{gathered}
\frac{\partial U(x, y)}{\partial x}=\frac{d U(\bar{z})}{d \bar{z}} \cdot \frac{\partial \bar{z}}{\partial x}=\frac{d U(\bar{z})}{d \bar{z}}=\mathfrak{R}\left[\frac{d \bar{W}(\bar{z})}{d \bar{z}}\right] \\
\frac{\partial V(x, y)}{\partial x}=\frac{d V(\bar{z})}{d \bar{z}} \cdot \frac{\partial \bar{z}}{\partial x}=\frac{d V(\bar{z})}{d \bar{z}}=\mathfrak{I}\left[\frac{d \bar{W}(\bar{z})}{d \bar{z}}\right] \\
\frac{\partial U(x, y)}{\partial y}=\frac{d U(\bar{z})}{d \bar{z}} \cdot \frac{\partial \bar{z}}{\partial y}=j \frac{d U(\bar{z})}{d \bar{z}} ; \frac{\partial V(x, y)}{\partial y}=\frac{d V(\bar{z})}{d \bar{z}} \cdot \frac{\partial \bar{z}}{\partial y}=j \frac{d V(\bar{z})}{d \bar{z}} .
\end{gathered}
$$

Replacing (45) in (43), it is obtained

$$
\frac{d \bar{W}(\bar{z})}{d \bar{z}}=\frac{\partial V(x, y)}{\partial y}-j \frac{\partial U(x, y)}{\partial y}
$$

$2 \mathfrak{R}[\cdot]$ and $\mathfrak{I}[\cdot]$ are, respectively, the real and imaginary parts of the arguments. 
From (46) and (44), it is easily shown that

$$
\frac{\partial V(x, y)}{\partial y}=\mathfrak{R}\left[\frac{d \bar{W}(\bar{z})}{d \bar{z}}\right] ; \quad \frac{\partial U(x, y)}{\partial y}=-\mathfrak{I}\left[\frac{d \bar{W}(\bar{z})}{d \bar{z}}\right] .
$$

It should be noticed that from (45) and (47) it results

$$
\frac{\partial U(x, y)}{\partial x}=\frac{\partial V(x, y)}{\partial y} ; \frac{\partial U(x, y)}{\partial y}=-\frac{\partial V(x, y)}{\partial x} .
$$

It is worth noticing that (48) corresponds to the Cauchy-Riemann condition, which states that, in fact, $\bar{W}(\bar{z})$ is an analytical function.

Given the initial guess $\left(x_{0}, y_{0}\right)$ the numerical iteration process starts. A new value is obtained using (41) and (42) taking into account (44) and (47) and it is used as the initial condition for the next iteration until the difference for the previous guess is within a predefined range (for example, less than $10^{-8}$ ). The method is very fast, while strongly dependent on the initial guess. Moreover, it assumes that the analytical description of the complex function $\bar{W}(\bar{z})$ is known. It can be a good option, whereas as the structure under analysis is of moderate complexity. Some examples are given below.

\subsection{Threshold analysis of anti-reflective (AR) coated conventional DFB lasers}

These lasers avoid the uncertainty related to the phase facets. Starting from (32) and assuming $\hat{r}_{1}=\hat{r}_{2}=0$, it yields

$$
j(\bar{\gamma} L)= \pm \bar{\kappa} \cdot L \cdot \sinh (\bar{\gamma} L) .
$$

There exist two pairs of possible solutions for each oscillation mode (mathematically those solutions correspond to complex conjugates). The solutions, gain and detuning related to the several modes that are allowed to propagate inside the cavity, are symmetrically placed related to the Bragg wavelength, where $\delta L=0$. Therefore, the laser spectrum is double degenerate. Since there is no solution with null detuning (Agrawall \& Dutta, 1986), the SLM operation is prevented. In spite of being the less complex DFB structure, it is useless in the OCS domain.

However, some remarks should be emphasized for this type of laser structures. For a given laser cavity, when the coupling coefficient increases, the normalized amplitude gain decreases or, equivalently, the threshold current will decrease. This is consistent with the fact that a larger coupling coefficient means a stronger optical feedback along the DFB laser structure. Alternatively, a reduction in the threshold gains can be obtained for a fixed coupling coefficient using a longer cavity length, since a larger single pass gain can be more easily achieved.

\subsection{Threshold analysis of AR-coated, single phase-shifted (1PS) DFB lasers}

As previously referred, a stable SLM operation is not guaranteed in conventional DFB lasers: neither in AR-coated DFB, since the laser spectra is double degenerate, nor in several reflective facets DFB lasers, due to the randomness of the corrugation phase at the laser facets. To overcome this drawback, some alterations should be included in the laser 
corrugation. The most popular solution corresponds to the inclusion of a single phase-shift discontinuity in the corrugation.

The laser characteristics are shown to be strongly dependent on the value assumed for the phase discontinuity and on its location inside the cavity (Ghafouri-Shiraz, 2003; Fernandes et al., 2009). It can be shown that one of the most advantageous situations corresponds to a phase-shift of $90^{\circ}$ placed near the centre of the cavity. This structure is referred as quarterlywavelength-shifted (QWS) and it is related to important improvements in the main laser figures of merit near threshold regime defined for OCS.

Based on the coupled-wave theory and after some tedious algebraic manipulations by matching all the boundary conditions (Ghafouri-Shiraz, 2003), the oscillating equation for an AR 1PS-DFB laser with a phase-shift discontinuity $\phi$ placed at the cavity centre is found, being given by $\left[\bar{\kappa} \hat{\Gamma}(1-\exp (\bar{\gamma} L)) /\left(\bar{\kappa}^{2}+\hat{\Gamma} \exp (\bar{\gamma} L)\right)\right]^{2}=\exp (2 j \phi)$ with $\hat{\Gamma}=\alpha-j \delta-\bar{\gamma}$.

\begin{tabular}{|c|c|}
\hline Laser structure & Complex equation \\
\hline AR- DFB & $j(\bar{\gamma} L)= \pm \bar{\kappa} \cdot L \cdot \sinh (\bar{\gamma} L)$ \\
\hline DFB with reflexive facets & $\begin{array}{c}(\bar{\gamma} L)^{2} \cdot D+(\bar{\kappa} \cdot L)^{2} \cdot \sinh ^{2}(\bar{\gamma} L) \cdot\left(1-\hat{r}_{1}^{2}\right) \cdot\left(1-\hat{r}_{2}^{2}\right)+j 2 . \\
\cdot(\bar{\kappa} \cdot L) \cdot\left(\hat{r}_{1}^{2}+\hat{r}_{2}^{2}\right) \cdot\left(1-\hat{r}_{1}^{2} \cdot \hat{r}_{2}^{2}\right) \cdot(\bar{\gamma} L) \cdot \sinh (\bar{\gamma} L) \cdot \cosh (\bar{\gamma} L)=0\end{array}$ \\
\hline AR-1PS DFB & {$\left[\bar{\kappa} \hat{\Gamma}(1-\exp (\bar{\gamma} L)) /\left(\bar{\kappa}^{2}+\hat{\Gamma} \exp (\bar{\gamma} L)\right]^{2}=\exp (2 j \phi)\right.$} \\
\hline FP & $\hat{r}_{1} \cdot \hat{r}_{2} \exp (-j \bar{k} L)=1$ \\
\hline
\end{tabular}

Table 1. Transcendental equations associated to oscillation conditions in some very simple laser structures.

Table 1 summarizes the equations assumed by the oscillation condition for the DFB lasers described in sections 2.1, 2.2 and 2.3. The first two cases correspond to conventional DFB lasers, i.e., those with perfect periodic corrugations. The first of them is AR-coated type, and it corresponds to (49); the second structure has finite reflectivity facets and it corresponds to (32). The third structure is an AR-coated DFB laser with a single phase-discontinuity $\phi$ placed in the middle of the cavity. The last row corresponds to a different type of laser: the Fabry-Pérot cavity, the simplest type of optical oscillator. There is no corrugation $(\bar{\kappa}=0)$ and the optical feedback that couples the two counter-running waves originates from the laser facets through their reflectivity values, $\hat{r}_{1}$ and $\hat{r}_{2}$.

\section{The static-TMM}

In section 2 the coupled wave theory has been applied to study the oscillation static conditions in several simple laser structures. Different eigen-value equations were obtained by matching different boundary conditions inside the laser cavity. From their solutions the oscillating modes in the cavity may be determined, from which the impacts due to laser parameters may be discussed.

Non-conventional DFB lasers diodes have been successively proposed to be used in OCS as improved alternatives to the QWS-DFB laser diode. These lasers aim to avoid the 
degradation of SLM operation with the current injection, by reducing the SHB effect (Agrawall \& Dutta, 1986; Ghafouri-Shiraz, 2003; Morthier \& Vankwikelberge, 1997). The SHB effect reduction can be achieved, for instance, by optimizing the coupling coefficient profile (Ghafouri-Shiraz, 2003) and/or modulating the corrugation pitch (Fessant, 1997) along the cavity length. However, the search for the improvement in the laser performance leads to the inclusion of additional boundary conditions that makes the static analysis of the modified laser structures based on the couple-wave theory tedious and inadequate, even for situations near the laser threshold regime, where non-linear effects are expected to be negligible.

More flexible methods are then required. It is generally accepted that TMM represents an adequate alternative to evaluate the laser performance in modified laser structures, as long as the included modifications are described in a matricial form. The great flexibility of the method relies on the fact that, in those assumptions, the same algorithm may be straightforward applied to the analysis of several laser structures.

\subsection{The threshold regime}

Basically, to perform the static-TMM-based model for the laser threshold analysis, the cavity with length $L$ is divided into $M$ concatenated sections, each one being identified by the constancy of its structural parameters. These are, for the $m$-th section with length $L_{m}$ : the corrugation period $\Lambda_{m}$, the amount of feedback per unit length $\bar{\kappa}_{m}$ and the phase of the section grating with respect to the left side of the section $\Omega_{m}$ (Fig. 2).

Each section is described by two counter-propagating electrical field waves, given by their complex amplitudes $\bar{E}_{R}(z)$ and $\bar{E}_{S}(z)$, which allow the internal electrical field intensity $\mathrm{E}(t, z)$ to be determined according to

$$
E(t, z) \propto \Re\left\{\left[\bar{E}_{R}(z)+\bar{E}_{S}(z)\right] \cdot \exp (j \omega t)\right\}=\Re\{[\bar{E}(z)] \cdot \exp (j \omega t)\} .
$$

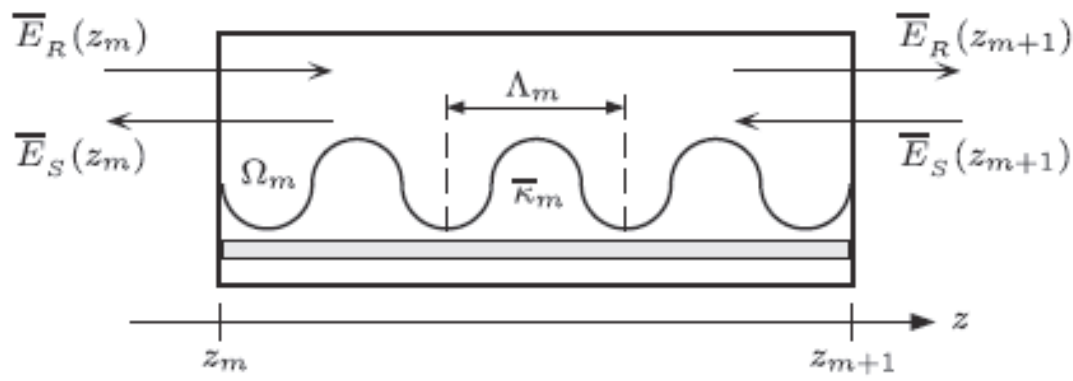

Fig. 2. A schematic diagram for a one-dimensional DFB laser structure section, placed between $z_{m}$ and $z_{m+1}$.

From (22), it is obtained

$$
\bar{E}(z)=\bar{E}_{R}(z)+\bar{E}_{S}(z)=\bar{R}(z) \cdot \exp \left(-j \cdot \beta_{m} \cdot z\right)+\bar{S}(z) \cdot \exp \left(j \cdot \beta_{m} \cdot z\right),
$$


where

$$
\begin{aligned}
& \bar{R}(z)=\bar{R}_{1 m} \cdot \exp \left(\bar{\gamma}_{m} \cdot z\right)+\bar{R}_{2 m} \cdot \exp \left(-\bar{\gamma}_{m} \cdot z\right) \\
& \bar{S}(z)=\bar{S}_{1 m} \cdot \exp \left(\bar{\gamma}_{m} \cdot z\right)+\bar{S}_{2 m} \cdot \exp \left(-\bar{\gamma}_{m} \cdot z\right),
\end{aligned}
$$

with

$$
\bar{S}_{1 m}=\bar{\rho}_{m} \cdot \exp \left(j \cdot \Omega_{m}\right) \cdot \bar{R}_{1 m} ; \bar{R}_{2 m}=\bar{\rho}_{m} \cdot \exp \left(-j \cdot \Omega_{m}\right) \cdot \bar{S}_{2 m}
$$

and

$$
\begin{gathered}
\bar{\rho}_{m} \triangleq \frac{j \bar{\kappa}_{m}}{\alpha-j \delta_{m}+\bar{\gamma}_{m}} ; \bar{\gamma}_{m} \hat{=} \sqrt{\left(\alpha-j \delta_{m}\right)^{2}+\bar{\kappa}_{m}^{2}} \\
\delta_{m} \triangleq \hat{=} \delta+\pi\left(\frac{1}{\Lambda_{1}}-\frac{1}{\Lambda_{m}}\right) ; \quad \beta_{m} \triangleq \frac{\pi}{\Lambda_{m}} \\
\Omega_{m}=\Omega_{1}+2 \sum_{k=1}^{m-1}\left(\frac{\pi}{\Lambda_{k}} L_{k}\right) ; 2 \leq m \leq M .
\end{gathered}
$$

In (54) $\alpha$ and $\delta$ are, respectively, the gain and the detuning, taking the left section as a reference. Using (53) in (52) it yields

$$
\left\{\begin{array}{l}
\bar{R}(z)=\bar{R}_{1 m} \cdot \exp \left(\bar{\gamma}_{m} \cdot z\right)+\bar{\rho}_{m} \cdot \bar{S}_{2 m} \cdot \exp \left(-j \cdot \Omega_{m}\right) \cdot \exp \left(-\bar{\gamma}_{m} \cdot z\right) \\
\bar{S}(z)=\bar{\rho}_{m} \cdot \bar{R}_{1 m} \cdot \exp \left(j \cdot \Omega_{m}\right) \cdot \exp \left(\bar{\gamma}_{m} \cdot z\right)+\bar{S}_{2 m} \cdot \exp \left(-\bar{\gamma}_{m} \cdot z\right)
\end{array} .\right.
$$

Assuming a generic $m$ cell, placed between $z=z_{m}$ and $z=z_{m+1}$, it is obtained from (56)

$$
\left\{\begin{array}{l}
\bar{R}\left(z_{m}\right)=\bar{R}_{1 m} \cdot \exp \left(\bar{\gamma}_{m} \cdot z_{m}\right)+\bar{\rho}_{m} \cdot \bar{S}_{2 m} \cdot \exp \left(-j \cdot \Omega_{m}\right) \cdot \exp \left(-\bar{\gamma}_{m} \cdot z_{m}\right) \\
\bar{S}\left(z_{m}\right)=\bar{\rho}_{m} \cdot \bar{R}_{1 m} \cdot \exp \left(j \cdot \Omega_{m}\right) \cdot \exp \left(\bar{\gamma}_{m} \cdot z_{m}\right)+\bar{S}_{2 m} \cdot \exp \left(-\bar{\gamma}_{m} \cdot z_{m}\right) \\
\bar{R}\left(z_{m+1}\right)=\bar{R}_{1 m+1} \cdot \exp \left(\bar{\gamma}_{m+1} \cdot z_{m+1}\right)+\bar{\rho}_{m+1} \cdot \bar{S}_{2 m+1} \cdot \exp \left(-j \cdot \Omega_{m+1}\right) \cdot \exp \left(-\bar{\gamma}_{m+1} \cdot z_{m+1}\right) \\
\bar{S}\left(z_{m+1}\right)=\bar{\rho}_{m+1} \cdot \bar{R}_{1 m+1} \cdot \exp \left(j \cdot \Omega_{m+1}\right) \cdot \exp \left(\bar{\gamma}_{m} \cdot z_{m+1}\right)+\bar{S}_{2 m+1} \cdot \exp \left(-\bar{\gamma}_{m+1} \cdot z_{m+1}\right)
\end{array} .\right.
$$

After an algebraic manipulation of (57) it is possible to write $\bar{R}\left(z_{m+1}\right)$ and $\bar{S}\left(z_{m+1}\right)$ as functions of $\bar{R}\left(z_{m}\right)$ and $\bar{S}\left(z_{m}\right)$. Finally, using (51) it is obtained

$$
\left[\begin{array}{c}
\bar{E}_{R}\left(z_{m+1}\right) \\
\bar{E}_{S}\left(z_{m+1}\right)
\end{array}\right]=\mathrm{T}\left(z_{m+1} / z_{m}\right) \cdot\left[\begin{array}{c}
\bar{E}_{R}\left(z_{m}\right) \\
\bar{E}_{S}\left(z_{m}\right)
\end{array}\right]
$$

where the transfer matrix for the $m$-th section of the one-dimensional DFB laser structure, $\mathrm{T}\left(z_{m+1} / z_{m}\right)$, links the column matrices related to the complex electric fields of the wave solutions at $z_{m}$ and $z_{m+1}$. It is given by 


$$
\mathrm{T}\left(z_{m+1} / z_{m}\right) \triangleq\left[\begin{array}{cc}
t_{11}^{m} & t_{12}^{m} \\
t_{21}^{m} & t_{22}^{m}
\end{array}\right],
$$

where $t_{11}^{m)}, t_{12}^{m)}, t_{21}^{m)}$ and $t_{22}^{m)}$ are given, respectively, by

$$
\begin{aligned}
& t_{11}^{m)} \triangleq \frac{\xi_{m}-\bar{\rho}_{m}^{2} \xi_{m}^{-1}}{\left(1-\bar{\rho}_{m}^{2}\right) \zeta_{m}} ; \quad t_{12}^{m)} \hat{=}-\frac{\bar{\rho}_{m}\left(\xi_{m}-\xi_{m}^{-1}\right) \cdot \exp \left(-j \Omega_{m}\right)}{\left(1-\bar{\rho}_{m}^{2}\right) \zeta_{m}}
\end{aligned}
$$

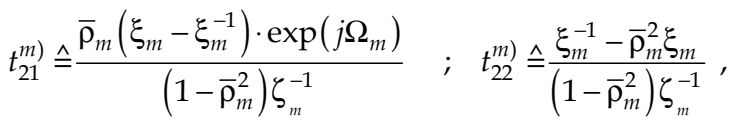

with $\xi_{m} \stackrel{\wedge}{=} \exp \left[\bar{\gamma}_{m}\left(z_{m+1}-z_{m}\right)\right]$ and $\zeta_{m} \hat{\wedge} \exp \left[j \beta_{m}\left(z_{m+1}-z_{m}\right)\right]$. Equations (58) to (60) are a generalization of the TMM presented in (Ghafouri-Shiraz, 2003), in order to allow the inclusion of variations in the grating period of laser structures, such as the CPM-DFB lasers. The fields at both cavity ends are connected by the elementary matrix product

$$
\left[\begin{array}{c}
\bar{E}_{R}(L) \\
\bar{E}_{S}(L)
\end{array}\right]=\left[\mathbf{T}_{\text {cor }}\right] \cdot\left[\begin{array}{l}
\bar{E}_{R}(0) \\
\bar{E}_{S}(0)
\end{array}\right],
$$

where

$$
\left[\mathbf{T}_{\text {cor }}\right] \triangleq \prod_{m=M}^{1} \mathrm{~T}\left(z_{m+1} / z_{m}\right) .
$$

Assuming that the field discontinuity is usually small along the plane of the phase-shift, the inclusion of one phase-shift $\varphi$ placed at $z=z_{m}$ (Fig. 3) may be described by the following set of equations

$$
\bar{E}_{R}\left(z_{m}^{+}\right)=\bar{E}_{R}\left(z_{m}^{-}\right) \cdot \exp (j \cdot \varphi) ; \bar{E}_{S}\left(z_{m}^{+}\right)=\bar{E}_{S}\left(z_{m}^{-}\right) \cdot \exp (-j \cdot \varphi) .
$$

The associated matrix is then given by

$$
\left[\mathbf{M}_{\boldsymbol{\varphi}}\right] \triangleq\left[\begin{array}{cc}
\exp (j \varphi) & 0 \\
0 & \exp (-j \varphi)
\end{array}\right]
$$

The matrix given by (64) should be included in the matrix product $\left[\mathbf{T}_{\text {cor }}\right]$ given by (62) at the correspondent $z$ position.

Let us now consider the cavity facet description. The uncertainty in the corrugation length regarding the period of the corrugation is itself a quantification of the uncertainty in the phase-shift related to the facet reflectivity. The left and right facet reflectivities are given, respectively, by

$$
\hat{r}_{1} \hat{=} r_{1} \cdot \exp \left(j \cdot \varphi_{1}\right) ; \quad \hat{r}_{2} \hat{=} r_{2} \cdot \exp \left(j \cdot \varphi_{2}\right)
$$


Fig. 4 represents schematically the counter-running waves at the left facet. Let us consider, firstly, the situation corresponding to $\varphi_{1}=\varphi_{2}=0$. For the left facet $(z=0)$, it yields

$$
\bar{E}_{R}\left(0^{+}\right)=\bar{E}_{R}\left(0^{-}\right) \cdot t_{1}+r_{1} \cdot \bar{E}_{S}\left(0^{+}\right) ; \quad \bar{E}_{S}\left(0^{-}\right)=\bar{E}_{S}\left(0^{+}\right) \cdot t_{1}-r_{1} \cdot \bar{E}_{R}\left(0^{-}\right) .
$$

In (66) $t_{1}$ is the left facet transmitivity. The second equation of (66) may be rewritten as

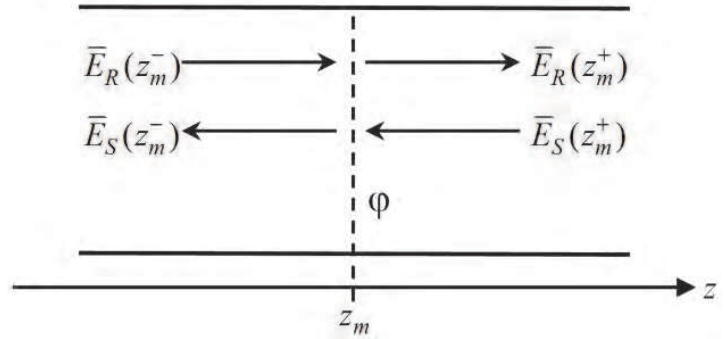

Fig. 3. A simplified schematic diagram of a phase-shift change $\varphi$ at $z=z_{m}$ in the DFB laser corrugation.

$$
\bar{E}_{S}\left(0^{+}\right)=\frac{1}{t_{1}} \cdot \bar{E}_{S}\left(0^{-}\right)+\frac{r_{1}}{t_{1}} \cdot \bar{E}_{R}\left(0^{-}\right) .
$$

Substituting (67) in the first equation of (66), it is obtained

$$
\bar{E}_{R}\left(0^{+}\right)=\bar{E}_{R}\left(0^{-}\right) \cdot\left(t_{1}+\frac{r_{1}^{2}}{t_{1}}\right)+\frac{r_{1}}{t_{1}} \cdot \bar{E}_{S}\left(0^{-}\right) .
$$

Assuming (67), (68) and that $t_{1}^{2}+r_{1}^{2}=1$, it results

$$
\left[\begin{array}{c}
\bar{E}_{R}\left(0^{+}\right) \\
\bar{E}_{S}\left(0^{+}\right)
\end{array}\right]=\frac{1}{t_{1}} \cdot\left[\begin{array}{cc}
1 & r_{1} \\
r_{1} & 1
\end{array}\right] \cdot\left[\begin{array}{c}
\bar{E}_{R}\left(0^{-}\right) \\
\bar{E}_{S}\left(0^{-}\right)
\end{array}\right] .
$$

Therefore, the matrix associated with the left facet, assuming $\varphi_{1}=0$, is given by

$$
\left[\mathbf{M}_{\mathbf{r}_{1}}\right] \triangleq \frac{1}{t_{1}} \cdot\left[\begin{array}{cc}
1 & r_{1} \\
r_{1} & 1
\end{array}\right]
$$

In order to include the phase associated with the left reflectivity we should consider a matrix associated with the phase- shift similar to (64). This means that

$$
\left[\begin{array}{c}
\bar{E}_{R}\left(0^{+}\right) \\
\bar{E}_{S}\left(0^{+}\right)
\end{array}\right]=\left[\mathbf{M}_{\varphi}\right] \cdot\left[\mathbf{M}_{\mathbf{r}_{1}}\right] \cdot\left[\begin{array}{c}
\bar{E}_{R}\left(0^{-}\right) \\
\bar{E}_{S}\left(0^{-}\right)
\end{array}\right]=\left[\begin{array}{cc}
\exp (j \varphi) & r_{1} \cdot \exp (j \varphi) \\
r_{1} \cdot \exp (-j \varphi) & \exp (-j \varphi)
\end{array}\right] \cdot\left[\begin{array}{c}
\bar{E}_{R}\left(0^{-}\right) \\
\bar{E}_{S}\left(0^{-}\right)
\end{array}\right] .
$$


In the oscillation condition the cavity incoming waves are null $\left(\bar{E}_{R}\left(0^{-}\right)=\bar{E}_{S}\left(L^{+}\right)=0\right)$, leading to

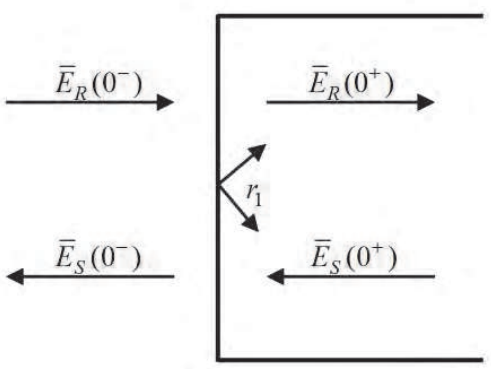

Fig. 4. A simplified schematic diagram of the two counter-running waves at left facet.

$$
\bar{E}_{R}\left(0^{+}\right)=\frac{1}{t_{1}} \cdot r_{1} \cdot \exp (j \varphi) \cdot \bar{E}_{S}\left(0^{-}\right) ; \bar{E}_{S}\left(0^{+}\right)=\frac{1}{t_{1}} \cdot \exp (-j \varphi),
$$

which originates

$$
\hat{r}_{1}=\frac{\bar{E}_{R}\left(0^{+}\right)}{\bar{E}_{S}\left(0^{+}\right)}=r_{1} \cdot \exp (2 \cdot j \cdot \varphi) .
$$

From (65) and (73) it results that $2 \cdot \varphi=\varphi_{1}$. Therefore

$$
\left.\left[\mathbf{M}_{\boldsymbol{\varphi}}\right] \triangleq \mathbf{M}_{\boldsymbol{\varphi}_{1}}\right]=\left[\begin{array}{cc}
\exp \left(j \frac{\varphi_{1}}{2}\right) & 0 \\
0 & \exp \left(-j \frac{\varphi_{1}}{2}\right)
\end{array}\right] .
$$

Similarly, it could be shown that

$$
\left[\mathbf{M}_{\mathbf{r}_{2}}\right]=\frac{1}{t_{2}} \cdot\left[\begin{array}{cc}
1 & -r_{2} \\
-r_{2} & 1
\end{array}\right] ; \quad\left[\mathbf{M}_{\varphi_{2}}\right]=\left[\begin{array}{cc}
\exp \left(j \frac{\varphi_{2}}{2}\right) & 0 \\
0 & \exp \left(-j \frac{\varphi_{2}}{2}\right)
\end{array}\right]
$$

The matrix $\left[\mathbf{T}_{\text {tot }}\right]$ for the overall cavity (corrugation+facets) will be then given by

$$
\left[\begin{array}{c}
\bar{E}_{R}\left(L^{+}\right) \\
\bar{E}_{S}\left(L^{+}\right)
\end{array}\right]=\left[\mathbf{M}_{\mathbf{r}_{2}}\right] \cdot\left[\mathbf{M}_{\mathbf{\varphi}_{2}}\right] \cdot\left[\mathbf{T}_{\mathrm{cor}}\right] \cdot\left[\mathbf{M}_{\mathbf{\varphi}_{1}}\right] \cdot\left[\mathbf{M}_{\mathbf{r}_{1}}\right] \cdot\left[\begin{array}{c}
\bar{E}_{R}\left(0^{-}\right) \\
\bar{E}_{S}\left(0^{-}\right)
\end{array}\right]=\left[\mathbf{T}_{\text {tot }}\right] \cdot\left[\begin{array}{c}
\bar{E}_{R}\left(0^{-}\right) \\
\bar{E}_{S}\left(0^{-}\right)
\end{array}\right],
$$

where $\left[\mathbf{T}_{\text {tot }}\right] \triangleq\left[\mathbf{M}_{\mathbf{r}_{2}}\right] \cdot\left[\mathbf{M}_{\boldsymbol{\varphi}_{2}}\right] \cdot\left[\mathbf{T}_{\text {cor }}\right] \cdot\left[\mathbf{M}_{\boldsymbol{\varphi}_{1}}\right] \cdot\left[\mathbf{M}_{\mathbf{r}_{1}}\right]$. Notice, that in modified DFB structures with axial variations of the coupling coefficient $\bar{\kappa}(z)$, the minimum number of sections to be 
considered in the static-TMM should be compatible with the assumption of a constant value for the coupling coefficient in each section. The oscillation condition corresponds to the vanishing of the incoming waves $\left(\bar{E}_{R}\left(0^{-}\right)=\bar{E}_{S}\left(L^{+}\right)=0\right)$. It is stated by the following requirement

$$
t_{22}^{\text {total }}(\alpha, \delta)=0 \text {, }
$$

where $t_{22}^{\text {total }}$ is the $4^{\text {th }}$ element of the matrix $\left[\mathbf{T}_{\text {tot }}\right]$. The solutions are the mode gain, $\alpha$, and the detuning, $\delta$, for each mode that is allowed to propagate inside the cavity. For the main mode their values are, respectively, the threshold gain, $\alpha_{t h}$, and the threshold detuning, $\delta_{t h}$. Considering a grating with a first-order Bragg diffraction, the mode gain and the detuning can be expressed, respectively, as (Ghafouri-Shiraz, 2003)

$$
\alpha(z)=\frac{\Gamma g(z)-\alpha_{\text {loss }}}{2} ; \delta(z)=\frac{2 \pi}{\lambda} n(z)-\frac{2 \pi \cdot n_{g}}{\lambda \cdot \lambda_{\Lambda}}\left(\lambda-\lambda_{\Lambda}\right)-\frac{\pi}{\Lambda(z)},
$$

where $\alpha_{\text {loss }}$ is the total loss, $n$ is the effective index, $\lambda$ is the lasing mode wavelength, $n_{g}$ is the group effective index and $g$ is the material gain, given by (Ghafouri-Shiraz, 2003)

$$
g(z)=A_{0}\left[N(z)-N_{0}\right]-A_{1}\left\{\lambda-\left[\lambda_{0}-A_{2}\left(N(z)-N_{0}\right)\right]\right\}^{2} .
$$

In (79), $N$ is the carrier concentration, $A_{0}$ is the differential gain, $N_{0}$ is the carrier concentration at transparency $(g=0), \lambda_{0}$ is the peak wavelength at transparency and $A_{1}$ and $A_{2}$ are parameters used in the parabolic model assumed for the material gain. Using the first-order approximation for the effective index $n$, one obtains (Ghafouri-Shiraz, 2003)

$$
n(z)=n_{0}+\Gamma \frac{\partial n}{\partial N} N(z)
$$

where $n_{0}$ is the effective index at zero carrier injection and $\partial n / \partial N$ is the differential index. The photon concentration $(S)$ and $N$ are coupled together through the steady-state carrier rate equation (Ghafouri-Shiraz, 2003)

$$
\frac{I}{q V_{a c t}}=A N(z)+B N^{2}(z)+C N^{3}(z)+\frac{v_{g} g(z) S(z)}{1+\varepsilon_{g} S(z)},
$$

where $I$ is the injection current, $q$ is the modulus of the electron charge, $V_{a c t}$ is the volume of the active layer, $A$ is the spontaneous emission rate, $B$ is the radiative spontaneous emission coefficient, $C$ is the Auger recombination coefficient, $\varepsilon_{g}$ is a non-linear coefficient that takes into account saturation effects and $v_{g}=c / n_{g}$ is the group velocity.

In a purely index-coupled DFB laser cavity, which is the case in the most of laser structures under analysis, the mutual interaction between the coupled waves can be neglected in the rate of total power change (Ghafouri-Shiraz, 2003; Kapon, et al., 1982). Therefore, the local photon density inside the cavity can be expressed as

$$
S(z) \approx \frac{2 \varepsilon_{0} n(z) n g \lambda}{h c} c_{0}^{2}\left[\left|\bar{E}_{R}(z)\right|^{2}+\left|\bar{E}_{S}(z)\right|^{2}\right]
$$


where $h$ is the Planck's constant, and $c_{0}$ a dimensionless coefficient that allows the determination of the total electric field at the above-threshold regime, taking into account that the normalization

$$
\left|\bar{E}_{R}(0)\right|^{2}+\left|\bar{E}_{S}(0)\right|^{2}=1
$$

has been imposed in the left cavity end. The boundary conditions at the left facet and (83) allow the calculation of the two counter-running waves, $\bar{E}_{R}(z)$ and $\bar{E}_{S}(z)$, at $z=0$. The use of the TMM allows the calculation of the longitudinal electric field profile. The output power at the right facet can therefore be determined as

$$
P=\frac{d w}{\Gamma} v_{g} \frac{h c}{\lambda} S(L)
$$

where $d$ and $w$ are the thickness and width of the active layer, respectively.

From the solutions of the oscillation condition (77), $\alpha_{t h}$ and $\delta_{t h}$ are determined. Using (78) to (80), the carrier concentration at threshold $\left(N_{t h}\right)$, the effective index at threshold $\left(n_{t h}\right)$, the threshold wavelength $\left(\lambda_{t h}\right)$, and $\lambda_{0}$ are successively evaluated. Threshold current $\left(I_{t h}\right)$ is then obtained from (81), assuming that $S$ is negligible at threshold. Within this assumption, the $z$ dependence is neglected in the first equation (78), (79) and (80). This is also true in the second equation (81), except for the CPM structures where a $z$ dependence should be included in $\Lambda(z)$.

The number $M$ of cells needed to implement the TMM-threshold analysis of several laser structures is summarized in Table 2.

\begin{tabular}{|c|c|c|c|}
\hline Laser structure & Number of cells $\boldsymbol{M}$ & $\begin{array}{c}\text { Number of Phase- } \\
\text { Shifts }\end{array}$ & Remarks \\
\hline FP & 3 & - & $\bar{\kappa}=0 ; \Lambda \rightarrow \infty$ \\
\hline AR-Conventional DFB & 1 & - & $\hat{r}_{1}=\hat{r}_{2}=0$ \\
\hline $\begin{array}{c}\text { Conventional DFB with } \\
\text { reflexive facets }\end{array}$ & 3 & 3 & $\hat{r}_{1}, \hat{r}_{2} \neq 0$ \\
\hline QWS & 5 & $N$ & $\hat{r}_{1}, \hat{r}_{2} \neq 0$ \\
\hline MPS & $2 N+3$ & - & $\hat{r}_{1}, \hat{r}_{2} \neq 0$ \\
\hline CPM & 5 & - & $\hat{r}_{1}, \hat{r}_{2} \neq 0$ \\
\hline CPM & Large number & - & $\hat{r}_{1}, \hat{r}_{2} \neq 0$ \\
\hline N layer VCSEL & $N+2$ & $\hat{r}_{1}, \hat{r}_{2} \neq 0$ \\
\hline
\end{tabular}

Table 2. Spatial discretization in static TMM for several semiconductor laser structures in the threshold regime.

The first conventional DFB structure is a mirrorless (AR) DFB laser. One single cell is needed for the corrugation description. The first CPM-DFB structure is a symmetric structure with two corrugation periods $\Lambda_{o}$ and $\Lambda_{c}$, respectively, for the outer zones, closer to the facets, 
and for the central zone. For the whole laser description five cells are needed: two cells for the facets, two cells for the outer zones in the corrugation and one cell for the central zone. The second CPM laser corresponds to a linear chirp corrugation, that is, a structure with a continuous change in the corrugation period.

\subsection{Above-threshold analysis}

In the above-threshold regime, $S(z)$ assumes high enough values to induce important nonuniformities in $N(z)$ and $n(z)$. Despite the SHB effect might be minimized by an adequate design of the DFB structure, the interdependence of $S(z), N(z)$ and $n(z)$ can't be neglected anymore. Therefore, in order to insure a correct evaluation of the above-threshold characteristics, each section shall be divided into several sub-sections. According to (Ghafouri-Shiraz, 2003), for a $500 \mu \mathrm{m}$ cavity length, about 5000 cells should be considered in order to ensure a reasonable accuracy in the stationary analysis.

The above-threshold calculations follow closely the method described in (Fessant, 1997; Ghafouri-Shiraz, 2003). However, in order to ensure a quick convergence in the evaluations of the laser characteristics, an adequate strategy is proposed.

\subsubsection{Lasing-mode analysis}

For each bias current $I$, the numerical above-threshold analysis concerning the lasing-mode is summarized as follows

a. Successive $(G \times G)$ grids are created in the $\left(c_{0}, \lambda\right)$ plane. The $i$-th grid is centered at $\left(c_{0_{c}}^{(i)}, \lambda_{c}^{(i)}\right)$ and it is enclosed in the region defined by the limits $c_{0_{\min }}^{(i)}, c_{0_{\max }}^{(i)}, \lambda_{\min }^{(i)}$ and $\lambda_{\max }^{(i)}$. For the initial grid $(i=1)^{3}$

$$
\begin{gathered}
\lambda_{c}^{(1)}=\lambda_{\text {th }} \\
c_{0_{c}}^{(1)}=\sqrt{\frac{h c\left(I-I_{t h}\right) /\left(2 q V_{a c t}{ }_{g} g_{t h} \varepsilon_{0} n_{t h} n_{g} \lambda_{t h}\right)}{\left|\bar{E}_{R}(0)\right|^{2}+\left|\bar{E}_{S}(0)\right|^{2}}}=\sqrt{\frac{h c\left(I-I_{t h}\right)}{2 q V_{a c t} v_{g} g_{t h} \varepsilon_{0} n_{t h} n_{g} \lambda_{t h}}} \\
c_{0_{\min }^{(1)}}^{(1)}=c_{0_{c}}^{(1)}-\Delta c_{0}^{(1)} ; \quad c_{0_{\max }}^{(1)}=c_{0_{c}}^{(1)}+\Delta c_{0}^{(1)} \\
\lambda_{\min }^{(1)}=\lambda_{c}^{(1)}-\Delta \lambda^{(1)} ; \quad \lambda_{\max }^{(1)}=\lambda_{c}^{(1)}+\Delta \lambda^{(1)} .
\end{gathered}
$$

For $G \simeq 10, \Delta c_{0}^{(1)} \triangleq c_{0_{c}}^{(1)} / 10$ and $\Delta \lambda^{(1)} \triangleq 0.1 \mathrm{~nm}$ seem adequate for most of DFB laser structures. However, a readjustment of $\Delta c_{0}^{(1)}$ and $\Delta \lambda^{(1)}$ may, occasionally, be necessary in order to prevent an eventual convergence towards a local minimum. This is a critical aspect of the proposed analysis, since an inadequate choice would prevent the numerical convergence.

b. For each one of the $G^{2}$ pairs of the $i$-th grid, $\left(c_{0_{k}}^{(1)}, \lambda_{l}^{(1)}\right)$ with $k ; l=1 \ldots G$, equations (7982) are self-consistently solved in order to determine the material gain, the carrier

\footnotetext{
${ }^{3}$ In (86) it has been taken into account the normalization condition (83).
} 
density, the photon density and the effective index for each one of the $j$-th sub-section, respectively, $g_{j}, N_{j}, S_{j}$ and $n_{j}$, with $1 \leq j \leq M$.

c. Equations (78) are solved in order to determine the lasing-mode gain and detuning for the $j$-th sub-section, respectively, $\alpha_{j}$ and $\delta_{j}$. The transfer matrix of the $j$-th sub-section , $\mathrm{T}\left(z_{j+1} / z_{j}\right)$ is then calculated.

d. Using the TMM, the two counter-running waves at the output of the $j$-th sub-section, $\bar{E}_{R_{j}}$ and $\bar{E}_{S_{j}}$, are obtained. For the $M$-th sub-section the discrepancy found between those values and the laser right facet boundary condition is represented by $\varepsilon_{k l}^{(i)}$. This value is evaluated and stored for each pair $\left(c_{0_{k}}^{(i)}, \lambda_{l}^{(i)}\right)$ of the $i$-th grid. The error associated to the $i$-th grid is given by $\varepsilon^{(i)}=\min \left(\varepsilon_{k l}^{(i)}\right)$.

e. Whenever $\varepsilon^{(i)}=\varepsilon^{(i-1)}$, the central pair remains the same $\left(c_{0_{c}}^{(i+1)}=c_{0_{c}}^{(i)}, \lambda_{c}^{(i+1)}=\lambda_{c}^{(i)}\right)$, but new limits are required for the next grid description. The partitions should be reduced considering, for instance: $\Delta c_{0}^{(i+1)}=\Delta c_{0}^{(i)} / 10$ and $\Delta \lambda^{(i+1)}=\Delta \lambda^{(i)} / 10$. Whenever $\varepsilon^{(i)}<\varepsilon^{(i-1)}$, the pair associated with $\min \left(\varepsilon_{k l}^{(i)}\right)$ is chosen as the next central pair $\left(c_{0_{c}}^{(i+1)}, \lambda_{c}^{(i+1)}\right)$, while $c_{0}$ and $\lambda$ partitions remain unchangeable. For $i=1, \varepsilon^{(i-1)}$ is taken as the error associated with the central pair $\left(c_{0_{c}}^{(1)}, \lambda_{c}^{(1)}\right)$.

For each one of the $G^{2}$ pairs $\left(c_{0_{k}}^{(i+1)}, \lambda_{l}^{(i+1)}\right)$, the steps a)-e) are repeated until $\varepsilon^{(i+1)}<\varepsilon_{\min }$, where $\varepsilon_{\min }$ is a preset error value, for instance, less than $10^{-14}$ (Ghafouri-Shiraz, 2003). Since the gain $\alpha_{j}$ and the detuning $\delta_{j}$ are $z$-dependent, the lasing characteristics for each bias current are associated with their mean values along the cavity, given by

$$
\alpha_{a v}(I)=\frac{1}{M} \sum_{j=1}^{M} \alpha_{j}(I) \quad ; \quad \delta_{a v}(I)=\frac{1}{M} \sum_{j=1}^{M} \delta_{j}(I) .
$$

Notice that the sequential analysis a) to e) assumes a one-mode propagation laser behavior. This procedure is itself a good assumption, since the present analysis focus on DFB structures that must guarantee SLM operation. Otherwise, different strategies should be adopted.

Finally, when studying the influence of the bias current on the laser characteristics, a considerable CPU time reduction can be achieved if, for each subsequent current, instead of using (85), $\lambda_{c}^{(1)}$ is taken as the solution found for the previous bias current.

\subsubsection{Side-mode analysis}

$S(z), N(z)$ and $n(z)$ profiles are settled for each bias current by the lasing-mode profiles obtained in section 3.2.1. At threshold, theses distributions are nearly uniform along the cavity, assuming average values, respectively, $0, N_{t h}$ and $n_{t h}$. The gain mode and detuning 
associated with the side-mode at threshold, respectively, $\alpha_{\text {side }}$ and $\delta_{1}$, are settled. In the one-mode approximation, the use of (79) leads to

$$
\lambda_{R}\left(\delta_{1}\right)=\frac{2 \pi \lambda_{B}\left(n_{t h}+n_{g}\right)}{\delta_{1} \lambda_{\Lambda}+2 \pi n_{g}+\frac{\pi \lambda_{\Lambda}}{\Lambda_{a v}}}
$$

where $\Lambda_{a v}$ is the average grating period given by

$$
\Lambda_{a v}=\frac{\sum_{m=1}^{M} L_{m} \cdot \Lambda_{m}}{L} .
$$

This assumption means that $\lambda_{R}\left(\delta_{1}\right)$ would be the threshold wavelength if $\delta_{1}$ would correspond to the lasing mode. On the other hand, regarding the side-mode gain, (78) imposes that

$$
2 \alpha_{\text {side }}=\Gamma g_{1}-\alpha_{\text {loss }}
$$

where $g_{1}$ is obtained from (82), making $N(z)=N_{\text {th }}$ and $\lambda=\bar{\lambda}_{1}\left(\alpha_{\text {side }}\right)$. The parameter $\bar{\lambda}_{1}\left(\alpha_{\text {side }}\right)$ should be interpreted as the wavelength in the one-mode approach if $\alpha_{\text {side }}$ would correspond to the threshold gain. It will be designated by the side-mode effective wavelength. Similarly, for the lasing mode, it is obtained

$$
2 \alpha_{t h}=\Gamma g_{t h}-\alpha_{\text {loss }}
$$

where $g_{t h}=A_{0}\left(N_{t h}-N_{0}\right)$. Then, from (91) and (92), it can be shown that

$$
\bar{\lambda}_{1}\left(\alpha_{\text {side }}\right)=\lambda_{\text {th }}+j \lambda_{I}\left(\alpha_{\text {side }}\right) ; \lambda_{I}\left(\alpha_{\text {side }}\right)=\sqrt{\frac{2\left(\alpha_{\text {side }}-\alpha_{\text {th }}\right)}{A_{1} \Gamma}} .
$$

A $(G \times G)$ grid is created in the plane $\left(\lambda_{I}, \lambda_{R}\right)$, adopting a similar procedure as the one described in Section 3.2.1 for the plane $\left(c_{0}, \lambda\right)$. The initial grid is centered in $\left(\lambda_{I_{c}}^{(1)}, \lambda_{R_{c}}^{(1)}\right)$, where $\lambda_{I_{c}}^{(1)}$ and $\lambda_{R_{c}}^{(1)}$ are given, respectively, by the second equation (93) and (89). The limits of the initial grid are defined by $\lambda_{I_{c}}^{(1)} \pm \Delta \lambda_{I}^{(1)}$ and $\lambda_{R_{c}}^{(1)} \pm \Delta \lambda_{R}^{(1)}$. The values $G=10, \Delta \lambda_{I}^{(1)} \simeq 0.01 \mathrm{~nm}$ and $\Delta \lambda_{R}^{(1)} \simeq 0.1 \mathrm{~nm}$ seem reasonable for most of the structures but, as previously referred, a readjustment may once in a while be necessary to avoid the mode hopping. Usually $\Delta \lambda_{I}^{(1)}$ is one order of magnitude lower than $\Delta \lambda_{R}^{(1)}$ because the difference between the normalized gains for different modes is about one order of magnitude lower than the difference between their normalized detunings.

Successive $(G \times G)$ grids are defined in the wavelength plane, centering the $i$-th grid in $\left(\lambda_{I_{c}}^{(i)}, \lambda_{R_{c}}^{(i)}\right)$, and enclosing it in the region defined by the limits $\lambda_{I_{c}}^{(i)} \pm \Delta \lambda_{I}^{(i)}$ and $\lambda_{R_{c}}^{(i)} \pm \Delta \lambda_{R}^{(i)}$. 
Then, for each bias current and pair $(k, l)$ of the $i$-th grid, i.e. $\left(\lambda_{I_{k}}^{(i)}, \lambda_{I_{l}}^{(i)}\right)$, the mode gain and detuning for each one of the $j(j=1, \ldots . M)$ sub-sections of the cavity are obtained as, respectively

$$
\alpha_{\text {side }_{k l_{j}}}^{(i)}=\alpha_{j}(I)+\left(\lambda_{I_{k}}^{(i)}\right)^{2} \frac{A_{1} \Gamma}{2} ; \delta_{\text {side }_{k l_{j}}}^{(i)}=\frac{2 \pi}{\lambda_{R_{l}}^{(i)}} n_{j}(I)-\frac{2 \pi n_{g}}{\lambda_{R_{l}}^{(i)} \lambda_{\Lambda}}\left(\lambda_{R_{l}}^{(i)}-\lambda_{B}\right)+\frac{\pi}{\Lambda_{j}} .
$$

In (95) $\alpha_{j}(I)$ and $n_{j}(I)$ are, respectively, the lasing-mode gain and the refractive index associated with the $j$-th subsection for a biasing current $I$ achieved in Section 3.2.1. Besides, $\Lambda_{j}$ is the corrugation period of the $j$-th subsection. Similarly as in Section 3.2.1, steps c)-e) are the sequentially followed. However, the side-mode analysis is quicker than the lasing mode analysis since the step b) is not implemented.

\section{The dynamic TMM}

In its conventional form, the transfer matrix $\mathrm{T}\left(z_{m+1} / z_{m}\right)$ of a given cell inside the laser cavity expresses the relationship described by (58). In this formulation, a steady-state operation has implicitly been assumed. It is now required to develop a time-dependent implementation of the TMM. As far as the dynamic-TMM is concerned, the increment of time requires updating the travelling-wave amplitudes as they pass through a section. The increment $\Delta t$ is chosen so that the spatial step size, $\Delta l$, is given by the product of the time increment by the group velocity $\left(\Delta l=\Delta t \times v_{g}\right)$. So, after one increment $\Delta t$, the backward wave $\bar{E}_{S}\left(z_{m+1}, t\right)$ travels one section to the left, becoming $\bar{E}_{S}\left(z_{m}, t+\Delta t\right)$, and the forward wave $\bar{E}_{R}\left(z_{m}, t\right)$ travels one section to the right, being then designated by $\bar{E}_{R}\left(z_{m+1}, t+\Delta t\right)$. Assuming that the transfer matrix remains unchanged during the time step, it yields after some simple manipulation of (58) (Lee et al., 1999) that

$$
\left[\begin{array}{l}
\bar{E}_{R}\left(z_{m+1}, t+\Delta t\right) \\
\bar{E}_{S}\left(z_{m}, t+\Delta t\right)
\end{array}\right]=\frac{1}{t_{22}^{(m)}}\left[\begin{array}{cc}
t_{11}^{(m)} t_{22}^{(m)}-t_{12}^{(m)} t_{21}^{(m)} & t_{12}^{(m)} \\
-t_{21}^{(m)} & 1
\end{array}\right] \times\left[\begin{array}{l}
\bar{E}_{R}\left(z_{m}, t\right) \\
\bar{E}_{S}\left(z_{m+1}, t\right)
\end{array}\right] .
$$

Equation (96) forms the basis of the dynamic TMM, where it is assumed that the variations in $\mathrm{T}\left(z_{m+1} / z_{m}\right)$ and in the wave amplitudes occur in a time scale negligible in comparison with the optical frequency. In a multi-electrode DFB model the local variations in carrier, photon and refractive index are taken into account by further dividing the separately pumped sections into subsections each one described by its own matrix $\mathrm{T}\left(z_{m+1} / z_{m}\right)$ (Davis \& O'Dowd, 1991, 1992). Obviously, accuracy increases with the number of cells, but it should always be kept in mind that the time computation increases almost quadratically with the number of cells: increasing $M$ decreases the step size $\Delta l$ and, simultaneously, the time increment $\Delta t$.

Dynamic-TMM analysis reinforces the relevance of the questions related to the need of decreasing the heavy simulation times arising from the intensive search for those laser parameters that complies with the boundary conditions of the problem under analysis. For 
typical lengths of hundred of micrometers and data rate less than about $40 \mathrm{~Gb} / \mathrm{s}$, simulation analysis requires no more than $M=100$ sections to guarantee enough method accuracy (Jia, X. et al., 2007). The model solves self-consistently the carrier and photon rate equations similarly as described in Section 3.1.

\section{Simulation results and discussions}

As an application example of the TMM it has been chosen a multiple phase-shift DFB laser structure especially designed to provide SLM operation.

\subsection{The laser structure}

The laser structure is represented in Fig. 5. It is a multi-section AR-coated DFB laser with uniform grating period $\left(\Lambda_{m}=\Lambda\right)$ and uniform coupling coefficient $\left(\bar{\kappa}_{m}=\bar{\kappa}\right)$. Three PS discontinuities $\left(\varphi_{i}\right)$ are located along the DFB laser structure. Their positions are represented by a normalized parameter given by

$$
\mathrm{PSP}_{i}=\frac{z_{i}}{L} \quad i=1,2,3,
$$

where $z_{i}$ is the $\varphi_{i}$ position.

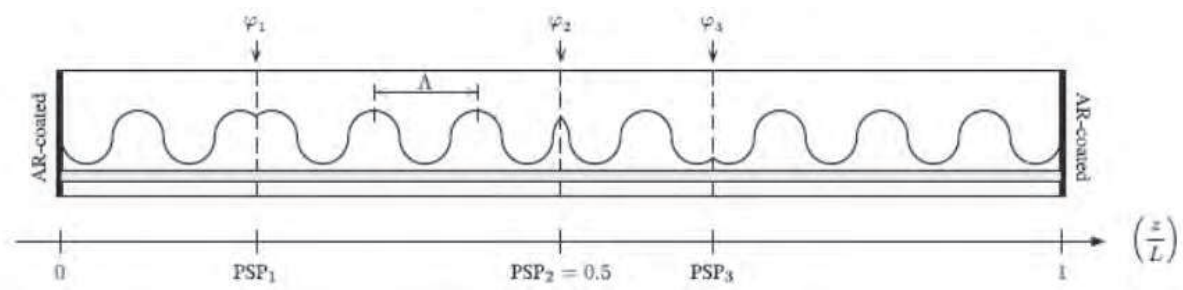

Fig. 5. A simplified schematic diagram of the 3PS-DFB laser structure with non-equal and non-identical 3PS.

A purely index-coupled laser structure assures that $\bar{\kappa}$ is real. For the structure, it has been assumed $L=500 \mu \mathrm{m}, \Lambda=227,039 \mathrm{~nm}, \Omega_{1}=0 \mathrm{rad}$ and $\mathrm{PSP}_{2}=0.5$. Two important laser figures of merit in the area of OCS are the normalized mode selectivity $\sigma$ and the flatness of the electric field distribution along the cavity $\mathfrak{I}$, which are given by

$$
\begin{aligned}
& \sigma=\alpha \cdot L-\alpha_{t h} \cdot L ; \\
& \mathfrak{I}=\frac{1}{L} \int_{0}^{L}\left[\mathrm{I}(z)-\mathrm{I}_{a v}\right]^{2} d z,
\end{aligned}
$$

where $\mathrm{I}(z)$ is the normalized electric field intensity at an arbitrary position $z$, which is given by

$$
\mathrm{I}(z)=\frac{\left|\bar{E}_{R}(z)\right|^{2}+\left|\bar{E}_{S}(z)\right|^{2}}{\left|\bar{E}_{R}(0)\right|^{2}+\left|\bar{E}_{S}(0)\right|^{2}}
$$


and $\mathbf{I}_{a v}$ is its average value along the cavity. Notice that according to the normalization condition (83), $\mathrm{I}(z)$ is numerically equal to $\left|\bar{E}_{R}(z)\right|^{2}+\left|\bar{E}_{S}(z)\right|^{2}$. For laser structures with $L=500 \mu \mathrm{m}$ it is generally accepted (Ghafouri-Shiraz, 2003) that a stable SLM operation requires $\sigma \geq 0.25$ and $\mathfrak{I} \leq 0.05$. The laser structural and material parameters used in the simulations are summarized in Table 3.

\begin{tabular}{|c|c|c|c|}
\hline Laser parameter & Value & Laser parameter & Value \\
\hline \multicolumn{2}{|c|}{ Material Parameters } & \multicolumn{2}{|c|}{ Structural parameters } \\
\hline Spontaneous emission rate, $A$ & $2.5 \times 10^{8} \mathrm{~s}^{-1}$ & Active layer width, $w$ & $1.5 \mu \mathrm{m}$ \\
\hline $\begin{array}{l}\text { Bimolecular recombination } \\
\text { coefficient, } B\end{array}$ & $1.0 \times 10^{-16} \mathrm{~m}^{3} \mathrm{~s}^{-1}$ & $\begin{array}{l}\text { Active layer thickness, } \\
d\end{array}$ & $0.12 \mu \mathrm{m}$ \\
\hline $\begin{array}{l}\text { Auger recombination } \\
\text { coefficient, } C\end{array}$ & $3.0 \times 10^{-41} \mathrm{~m}^{6} \mathrm{~s}^{-1}$ & Cavity length, $L$ & $500 \mu \mathrm{m}$ \\
\hline Differential gain, $A_{0}$ & $2.7 \times 10^{-20} \mathrm{~m}^{2}$ & $\begin{array}{l}\text { Optical confinement } \\
\text { factor, } \Gamma\end{array}$ & 0.35 \\
\hline Gain curvature, $A_{1}$ & $1.5 \times 10^{19} \mathrm{~m}^{-3}$ & Grating period, $\Lambda$ & $227.039 \mathrm{~nm}$ \\
\hline $\begin{array}{l}\text { Differential peak wavelength, } \\
A_{2}\end{array}$ & $2.7 \times 10^{-32} \mathrm{~m}^{4}$ & & \\
\hline Internal loss, $\alpha_{\text {loss }}$ & $4.0 \times 10^{3} \mathrm{~m}^{-1}$ & & \\
\hline $\begin{array}{l}\text { Effective index at zero } \\
\text { injection, } n_{0}\end{array}$ & 3.41351524 & & \\
\hline $\begin{array}{l}\text { Carrier density at } \\
\text { transparency, } N_{0}\end{array}$ & $1.23 \times 10^{24} \mathrm{~m}^{-3}$ & & \\
\hline Differential index, $d n / d N$ & $-1.8 \times 10^{-26} \mathrm{~m}^{3}$ & & \\
\hline Group velocity, $v_{g}$ & $8.33 \times 10^{7} \mathrm{~m} \times \mathrm{s}^{-1}$ & & \\
\hline Nonlinear gain coefficient, $\varepsilon_{g}$ & $1.5 \times 10^{-23} \mathrm{~m}^{3}$ & & \\
\hline
\end{tabular}

Table 3. Summary of laser parameters.

\subsection{The structure optimization (threshold situation)}

The objective is twofold: to maximize $\sigma$ and to minimize $\mathfrak{I}$ at threshold. For this purpose, it will be varied, simultaneously and independently, the following set of variables (decision variables): $\bar{\kappa} L, \varphi_{2}, \mathrm{PSP}_{1}, \varphi_{1}, \mathrm{PSP}_{3}$ and $\varphi_{3}$. The procedure initializes with the boundary values that insure a stable SLM operation according to the selection criteria previously referred, i.e., $\sigma=\sigma_{\min }=0.25$ and $\mathfrak{I}=\mathfrak{I}_{\max }=0.05$. After each step, these values are adjusted by fixing tighter limits, i.e., higher $\sigma$ and smaller $\mathfrak{I}$. The starting point is a AR QWS-DFB laser structure $4\left(\varphi_{1}=0, \varphi_{2}=90^{\circ}, \varphi_{3}=0\right)$ with $\bar{\kappa} L=2$. In the specialized literature these

${ }^{4}$ This phase change corresponds to a quarter wavelength shift and so, the name single $\lambda / 4$-shifted DFB also used. 
lasers are often associated with high mode selectivity, zero frequency and small current density at threshold. Nevertheless, the highly non-uniform electric field distribution induces local carrier depletion near the centre of the cavity that is responsible for the degradation of the laser performance in the high power regime. In the procedure adopted hereby it will always be assumed that $\mathrm{PSP}_{1} \leq \mathrm{PSP}_{2}$ and $\mathrm{PSP}_{2} \leq \mathrm{PSP}_{3}$. The step-by-step procedure can be summarized as follows

Step 1. One PS, $\left(\varphi_{1}\right)$, is added in the first half of the cavity. The optimization of $\sigma\left(\mathrm{PSP}_{1}, \varphi_{1}\right)$ and $\mathfrak{I}\left(\mathrm{PSP}_{1}, \varphi_{1}\right)$ is performed by varying simultaneously and independently both arguments in their ranges: $0 \leq \mathrm{PSP}_{1} \leq 0.5$ and $0^{\circ} \leq \varphi_{1} \leq 180^{\circ}$. It will be assumed as selection criteria that $\sigma\left(\operatorname{PSP}_{1}, \varphi_{1}\right) \geq \sigma_{\min }$ and $\mathfrak{I}\left(\mathrm{PSP}_{1}, \varphi_{1}\right) \leq \mathfrak{I}_{\max }$. This procedure will lead to the definition of a region in the $\left(\mathrm{PSP}_{1}, \varphi_{1}\right)$ plane from which a solution is chosen and new boundaries $\left(\sigma_{\min }, \mathfrak{I}_{\max }\right)$ are settled;

Step 2. For the new boundaries, another PS, $\left(\varphi_{3}\right)$, is placed in the second half of the cavity. A similar procedure as the one described in step 1 is adopted, now for $\sigma\left(\mathrm{PSP}_{3}, \varphi_{3}\right)$ and $\mathfrak{I}\left(\mathrm{PSP}_{3}, \varphi_{3}\right)$, assuming $0.5 \leq \mathrm{PSP}_{3} \leq 1$ and $0^{\circ} \leq \varphi_{3} \leq 180^{\circ}$.

Steps 1 and 2 are sequentially repeated until no improvements on $\sigma$ and $\mathfrak{I}$ are achieved. The optima values for the set $\left(\mathrm{PSP}_{1}, \varphi_{1}, \mathrm{PSP}_{3}, \varphi_{3}\right)$ are found, assuming $\bar{\kappa} L=2$ and $\varphi_{2}=90^{\circ}$. New optima boundaries $\left(\sigma_{\min }, \mathfrak{I}_{\max }\right)$ are settled.

Step 3. An optimization of $\sigma\left(\bar{\kappa} L, \varphi_{2}\right)$ and $\mathfrak{I}\left(\bar{\kappa} L, \varphi_{2}\right)$ is performed by varying simultaneously and independently both arguments in their ranges: $1 \leq \bar{\kappa} L \leq 3$ and $0^{\circ} \leq \varphi_{2} \leq 180^{\circ}$. It will be assumed as selection criteria that $\sigma\left(\bar{\kappa} L, \varphi_{2}\right) \geq \sigma_{\min }$ and $\mathfrak{I}\left(\bar{\kappa} L, \varphi_{2}\right) \leq \mathfrak{I}_{\max }$

Steps 1, 2 and 3 are repeated until no improvements on $\sigma$ and $\mathfrak{I}$ are achieved. This means that the best 3PS-DFB laser structure $\left(\mathrm{PSP}_{1}, \varphi_{1}, \mathrm{PSP}_{2}=0.5, \varphi_{2}=90^{\circ}, \mathrm{PSP}_{3}, \varphi_{3}, \overline{\mathrm{\kappa}} L\right)$ is obtained, as far as $\sigma$ and $\mathfrak{I}$ are concerned. In all steps, and whenever necessary, an argument based on the smallest threshold gain is used in order to decide the best solution.

Fig.6 and Fig.7 show, respectively, the contour maps for $\sigma\left(\mathrm{PSP}_{3}, \varphi_{3}\right)$ and $\mathfrak{I}\left(\mathrm{PSP}_{3}, \varphi_{3}\right)$, when the arguments vary along their entire range, assuming $\mathrm{PSP}_{1}=0.127, \varphi_{1}=110.7^{\circ}, \mathrm{PSP}_{2}=0.5, \varphi_{2}=60^{\circ}$ and $\bar{\kappa} L=1.7$.

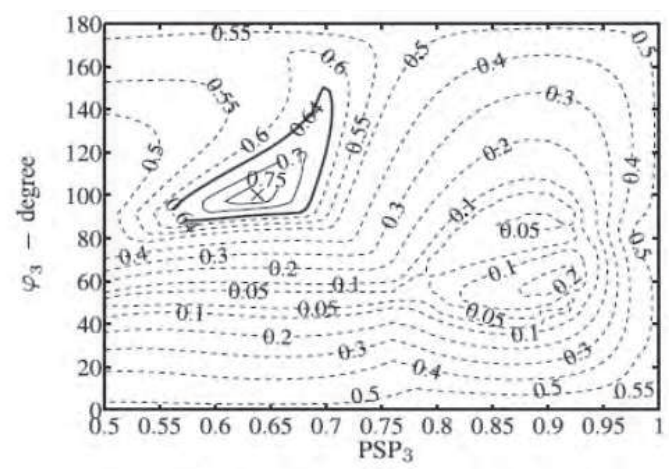

Fig. 6. Contour maps of the mode selectivity in the $\left(\mathrm{PSP}_{3}, \varphi_{3}\right)$ plane. Values for $\sigma \geq 0.64$ are represented by solid lines. 


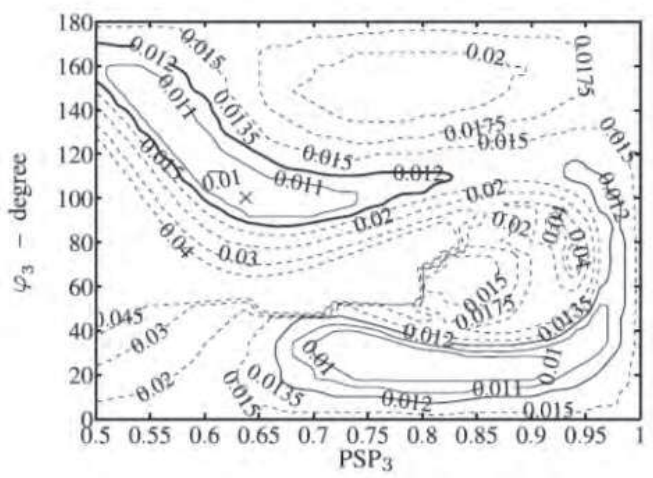

Fig. 7. Contour maps of the flatness in the $\left(\mathrm{PSP}_{3}, \varphi_{3}\right)$ plane. Values for $\mathfrak{I} \leq 0.012$ are represented by solid lines.

Solid lines enclose all combinations $\left(\mathrm{PSP}_{3}, \varphi_{3}\right)$ that ensure $\sigma\left(\bar{\kappa} L, \varphi_{2}\right) \geq 0.64$ and $\mathfrak{I}\left(\bar{\kappa} L, \varphi_{2}\right) \leq 0.012$, since $\sigma_{\min }=0.64$ and $\mathfrak{I}_{\max }=0.012$ have been settled in the previous iteration. Within all the possibilities, the chosen solution, $(x)$, is $\left(\mathrm{PSP}_{3}=0.64, \varphi_{3}=100^{\circ}\right)$, which corresponds to $\sigma_{\min }=0.78$ and $\mathfrak{I}_{\max }=0.010$. At the end of the optimization process, the final solution has been found: $\mathrm{PSP}_{1}=0.127, \varphi_{1}=110.7^{\circ}, \mathrm{PSP}_{2}=0.5, \varphi_{2}=60^{\circ}$, $\mathrm{PSP}_{3}=0.64, \varphi_{2}=100^{\circ}$ and $\overline{\mathrm{K}} L=1.7$. Besides, the optimized laser structure presents $\alpha_{t h}=1.18$, which corresponds to $I_{t h}=23.4 \mathrm{~mA}$. This value is similar to those reported in (Ghafouri-Shiraz, 2003) for the QWS-DFB and the symmetric 3PS-DFB lasers, respectively, $I_{\text {th }}=19.8 \mathrm{~mA}$ and $I_{\text {th }}=21.8 \mathrm{~mA}$.

Table 4 summarizes the results for $\sigma, \mathfrak{I}$ and $\alpha_{t h} L$ achieved for three different laser structures: the optimized 3PS-DFB (asymmetric), the QWS-DFB and the symmetric 3PS-DFB referred in (Ghafouri-Shiraz, 2003). All lasers are AR-type because the random corrugation phases at the laser facets will cause extra difficulty in controlling the laser characteristics.

\begin{tabular}{|c|c|c|c|}
\hline Laser structure & $\sigma$ & $\mathfrak{I}$ & $\alpha_{t h} L$ \\
\hline Asymmetric 3PS-DFB (optimized) & & & \\
PSP $_{1}=0.127 ; \varphi_{1}=110.7^{\circ}$ & 0.78 & 0.010 & 1.18 \\
PSP $_{2}=0.500 ; \quad \varphi_{2}=60^{\circ}$ & & & \\
PSP $_{3}=0.640 ; \varphi_{3}=100^{\circ}$ & & & 0.70 \\
\hline QWS-DFB & 0.73 & 0.30 & \\
\hline 3PS-DFB (Ghafouri-Shiraz, 2003) & & & 0.78 \\
PSP $_{1}=0.25 ; \quad \varphi_{1}=60^{\circ}$ & 0.34 & 0.012 & \\
PSP $_{2}=0.50 ; \varphi_{2}=60^{\circ}$ & & & \\
PSP $_{3}=0.75 ; \quad \varphi_{3}=60^{\circ}$ & & & \\
\hline
\end{tabular}

Table 4. Figures of merit for the symmetric, asymmetric 3PS-DFB and QWS-DFB laser structures. 
As far as the flatness is concerned, the 3PS-DFB laser structures are clearly advantageous. This is not surprising, since the inclusion of several PS along the laser cavity flattens the field distribution. However, it is worth noticing that the asymmetric 3PS-DFB structure reaches higher mode selectivity than the other two laser structures.

A threshold analysis has been presented. Nevertheless, one should always bear in mind that the results for a structure presenting an adequate performance at threshold are not conclusive. An above-threshold analysis is essential in order to assess the rate at which the $\mathrm{SHB}$ effect deteriorates the laser features with the increasing current.

\subsection{The above-threshold analysis}

We shall begin with the stationary analysis, but, as we shall refer later, the transient aspects may be determinant, which in fact imposes the need of a dynamic analysis in order to describe adequately the laser performance in the domain of high currents. Both analysis will lead to heavier simulations than the threshold analysis, since the number of cells needed for a correct evaluation of the carrier and photon profiles is deeply increased.

\subsubsection{The static-TMM results}

Fig. 8 shows the photon distribution in the asymmetric structure for different bias currents. It shows the gradual increase of the photon number in the whole structure, due to the stimulated emission. The 3PS-DFB lasers include local maxima other than the central one, leading to flatter distributions than those obtained for the QWS structure. Moreover, both 3PS-DFB lasers show smaller differences between the central photon density and the escaping photon densities at the facets, thus benefitting the laser performance as far as the emitted power is concerned, as it shall be seen later in the light-current stationary characteristics of these structures (Fig. 12).

Fig.9 shows the laser mode selectivity vs. current injection. Similar mode discriminations at threshold for the asymmetric 3PS and the QWS at threshold can be seen. Nevertheless, the mode selectivity has a severe reduction with increasing bias current for the QWS case, showing that the laser is strongly affected by the SHB effect. For the symmetric 3PS-DFB laser, it is apparent that the stability related to flatter photon profiles was obtained at an expense of a great reduction in the mode selectivity, while the situation is reverted at high values of biasing currents. Undoubtedly, the best option is the asymmetric 3PS optimized structure.

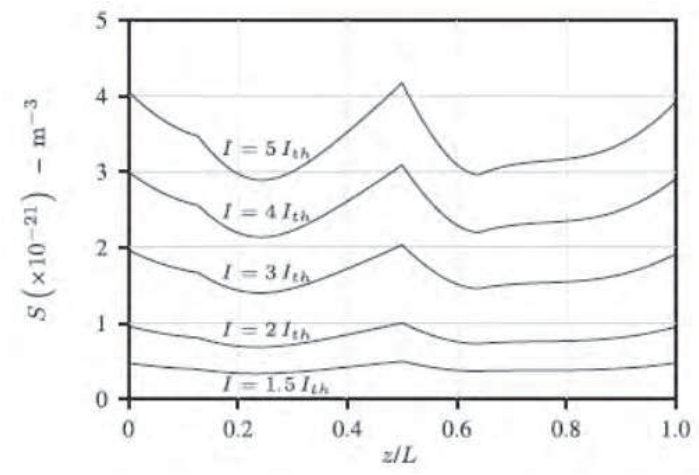

Fig. 8. $S(z)$ in the optimized asymmetric 3PS-DFB laser structure under different biasing currents. 


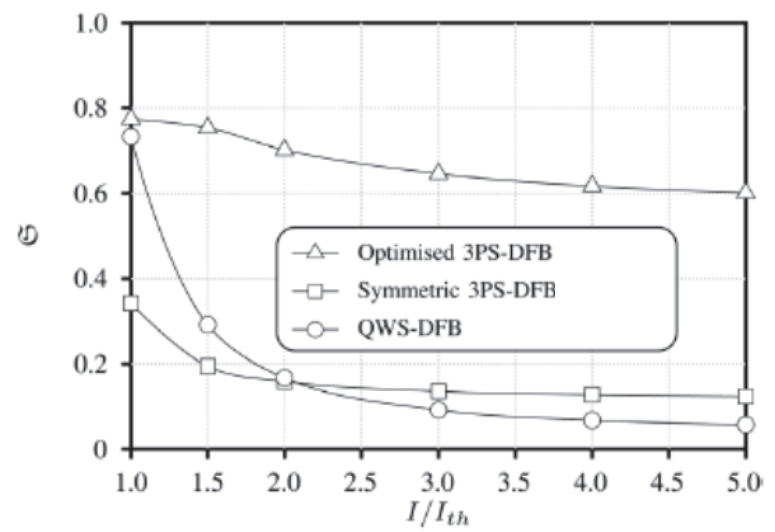

Fig. 9. Mode selectivity vs. current injection for the 3 structures under analysis.

Fig.10 focuses on the evolution of the flatness with current injection for the two 3PS-DFB lasers, showing a monotonically decreasing function for both structures. It should be emphasized that the flatness lies in the range defined by the selection criteria for both lasers, which is not definitely the case for the QWS-DFB, since this structure presents high-non uniformities in the photon profile $\left(\mathfrak{I}=0.3\right.$ at threshold, and $\mathfrak{I}=0.079$ for $\left.I=5 \times I_{t h}\right)$. Notice that the QWS-DFB laser flatness falls outside the axis limits.

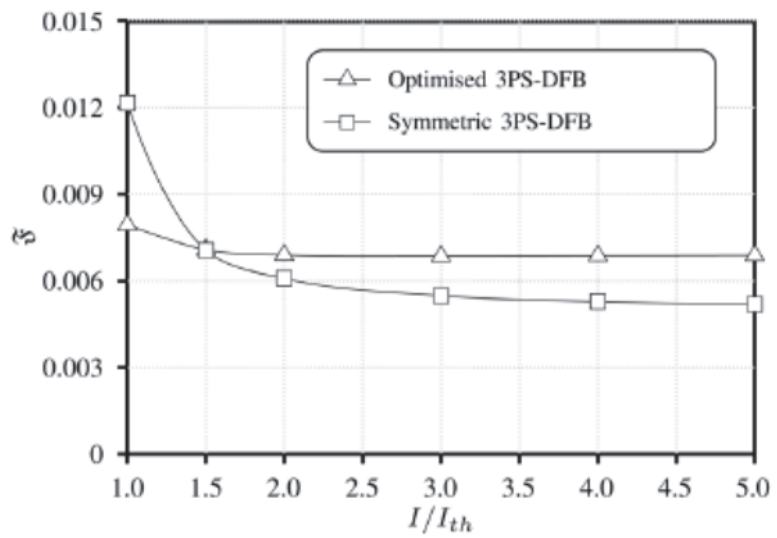

Fig. 10. Flatness vs. current injection for the three laser structures under analysis.

A comparative analysis of the three laser structures may be observed in Fig.11 to Fig.14, as far as the emitted power and wavelength are concerned. In the current range $1 \leq I / I_{t h} \leq 5$, relative variations in the emitted wavelengths $\left(\Delta \lambda / \lambda_{\text {th }}\right)$ of $9.4 \times 10^{-4} \%, 1.5 \times 10^{-3} \%$ and $5.5 \times 10^{-3} \%$ are observed for the asymmetric, the symmetric and the QWS lasers, respectively (Fig.11). Under similar normalized current injections the asymmetric structure shows larger values for the optical output power, measured at the right facet (Fig.12). This may be explained by the increase of the escaping photon density at right facet related to the induced 
asymmetry. A similar consequence would be attained using different facet reflectivities at the laser cavity ends (Boavida, et al., 2011).

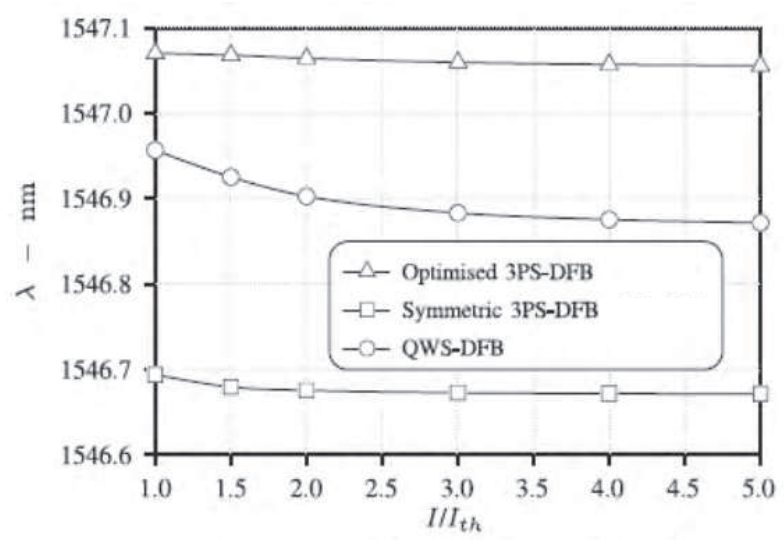

Fig. 11. Lasing wavelength $v s$ current injection for the 3 laser structures under analysis.

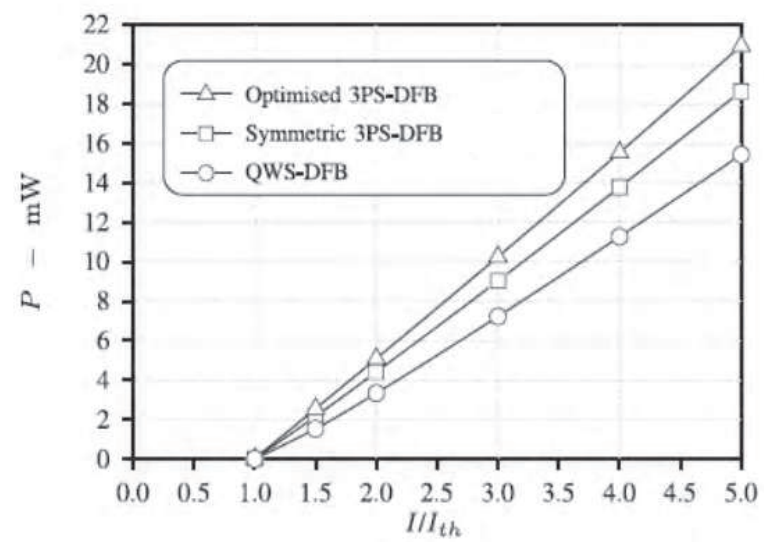

Fig. 12. Emitted power vs current injection for the 3 laser structures under analysis.

The measurement of the laser spectral characteristics is a way of checking its single-mode stability. Fig.13 shows the normalized spontaneous emission power for $I=1.5 \times I_{t h}$ and $I=5 \times I_{t h}$, for the asymmetric laser. High values are obtained for the side-mode-suppression ratio (SMSR) for both currents. Besides, it is worth noticing that the "blue-shift" in wavelengths is negligible. The inset of Fig. 10 shows the $\alpha(\delta)$ plot for the modes in the cavity at threshold. The figure points out two possible side-modes that are very close in frequency (encircled by a dashed line), which originates the broadening of the spectrum around $1546.4 \mathrm{~nm}$. Another relevant aspect lies with the fact that, near $1547.7 \mathrm{~nm}$, the spectral amplitude of the dominant mode remains at a high value when the current injection increases, showing no severe mode competition in the high power regime. 


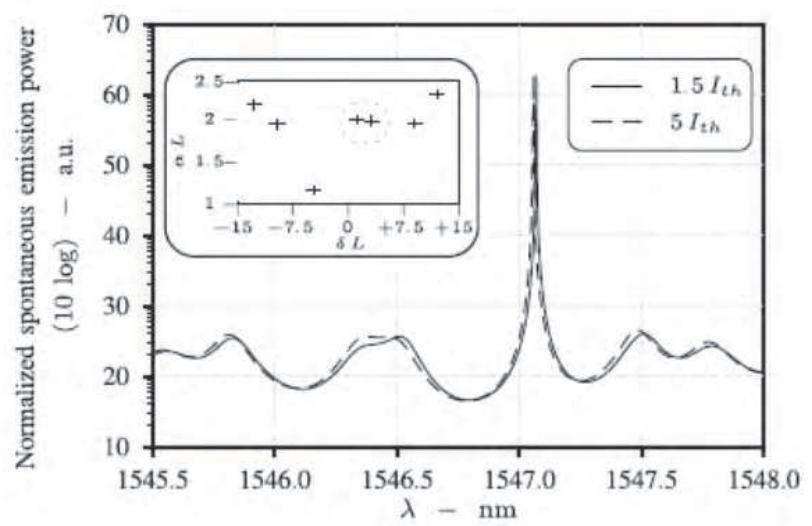

Fig. 13. Above-threshold normalized spontaneous emission spectra under two different biasing current for the asymmetric 3PS-DFB laser structure.

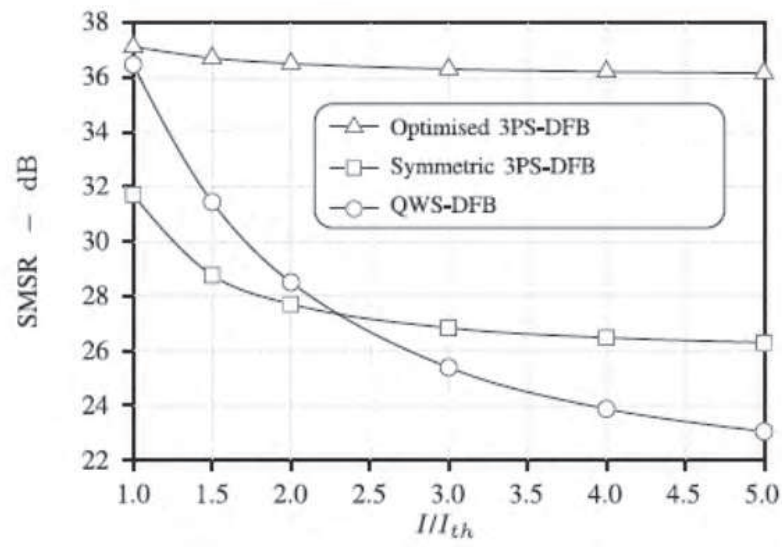

Fig. 14. Side-mode-suppression ratio vs current injection for the 3 laser structures under analysis.

This is pin-pointed in Fig.14, where the SMSR of the asymmetric structure is maintained throughout the range of biasing currents under analysis. This is not the case with the two other structures, the SMSR becoming lower than the required 30dB for the SLM operation (Morthier \& Vankwikelberge, 1997) over the most part of the current range. As we shall see in next section this will be enhanced in the results obtained from the dynamic-TMM.

\subsubsection{The dynamic-TMM results}

Fig. 15 illustrates the transient response (emitted power) of the asymmetric 3PS-DFB laser when $I$ is a step-function of $2 \times I_{t h}$. There is a delay of about $0.25 \mathrm{~ns}$ in the output $S(t)$ dynamics and a frequency of the relaxation oscillations of about $4 \mathrm{GHz}$, which agrees with the result obtained from the approximate expression (Agrawall \& Dutta, 1986) 


$$
f_{n} \cong \frac{1}{2 \pi} \sqrt{\frac{A_{0} v_{g} \Gamma\left(I-I_{t h}\right)}{q L w d}} .
$$

This parameter is usually known as the $-3 \mathrm{~dB}$ modulation bandwidth. Using the dynamic-TMM for step-like biasing currents, the stationary values can be interpreted as the asymptotic values of the time evolutions.

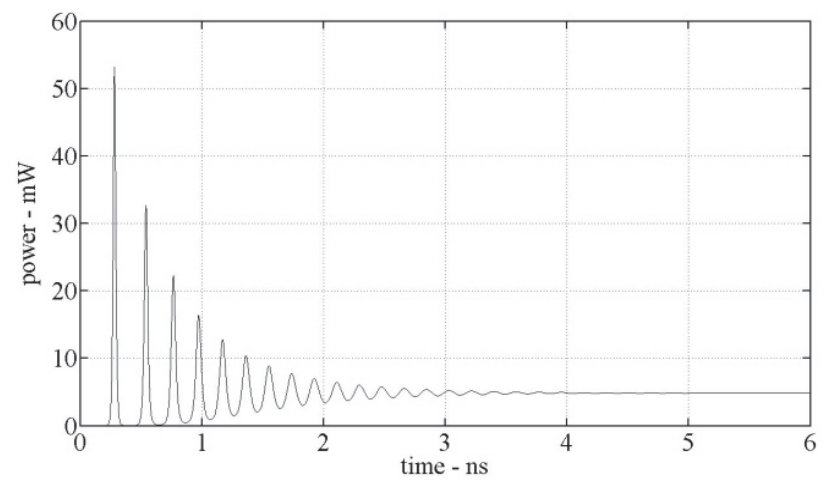

Fig. 15. Transient response of the asymmetric 3PS-DFB laser when the final current is $2 \times I_{\text {th }} \cong 46.8 \mathrm{~mA}$.

Fig. 16 compares the light-current characteristics obtained from the static-TMM with those extracted from the time evolutions obtained using the dynamic-TMM for the three lasers under analysis. Small deviations between the results obtained with the two TMM models are visible for the asymmetric 3PS-DFB laser, especially for high bias current values. This is due to the great difference in the number of sections that are present in the two models: 5000 cells in the static-TMM and only 100 cells in the dynamic-TMM. This may be especially important for the asymmetric structure, since using less than 1000 cells we cannot accurately define the first PS position in the asymmetric 3PS-DFB laser ( $\left.\mathrm{PSP}_{1}=0.127\right)$. However, it must always be kept in mind that

- As referred in Section 4 the time of computation increases almost quadratically with the number $M$ of cells;

- In order to obtain the stationary situation, time evolutions during 1-2 carrier lifetimes $\left(\tau_{n}\right)$ should be considered, where

$$
\tau_{n} \cong\left(A+B \cdot N_{t h}+C \cdot N_{t h}^{2}\right)^{-1}
$$

- For high currents, the lasing output of unstable lasers experiences transient oscillations that originates from the beating frequency of multiple mode lasing.

This last aspect is referred in (Jia et al., 2007) for the QWS-DFB laser in the transient response to a step-like biasing current whose final state value is $I=90 \mathrm{~mA}$. The oscillations in the emitted power arise from beating frequency of multiple mode lasing. The random feature of spontaneous emission is determinant in the transient response of the lasers, especially when the SMSR and the mode selectivity are small, which is the case for the QWS-DFB at high biasing currents. Its influence may be taken into account 
including Langevin noise sources as additional terms in the rate equations (Coldren \& Corzine, 1995). These sources are assumed to be white noise and are small enough to make use of the differential rate equations. The influence of spontaneous emission may be included in the TMM considering extra photon fluxes emerging from each cell to seed the growth of the travelling waves (Davis \& Dowd, 1992). Their influence is negligible in DFB lasers that guarantee sufficiently high SMSR in the high power regime. Therefore, since the side-mode is almost completely suppressed in the asymmetric 3PS-DFB, a good dynamic SLM operation is ensured.

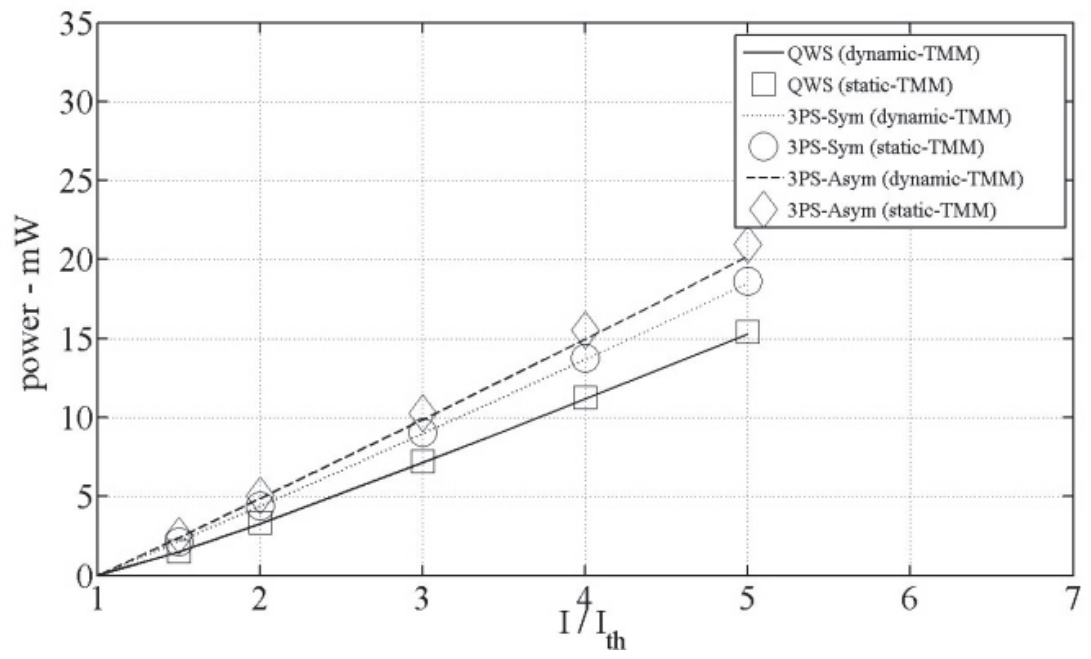

Fig. 16. Light-current stationary characteristics for lasers under analysis obtained using the static and the dynamic TMM.

\section{Conclusion}

The TMM is described both for static and dynamic analysis. The advantages of the TMM are numerous. Namely: it is not necessary to solve the coupled-mode equations, but instead we need to describe any perturbation in the wave propagation inside the laser cavity by the appropriate transfer matrix. This means that the same model works for several laser structures: FP, DFB, DBR (Kim \& Jeong, 2003) or any combination of these; external feedback is easily included; weak or strong coupling can be treated. The model can handle laser amplifiers as well.

The static-TMM has been used for the optimization of a multiple-phase-shifted DFB laser. Above-threshold analysis using both the static-TMM and the dynamic-TMM have demonstrated that indeed the main laser figures of merit of the 3PS-DFB optimized structure exceeded those for the commonly referred QWS-DFB or for other similar multiple-phaseshifted DFB structures presented elsewhere. We may conclude that the TMM, both in its static and dynamic versions, represents itself a powerful tool to be used in the important domain of OCS for the optimization of laser structures especially designed to provide SLM operation. 


\section{References}

Agrawall, G. \& Dutta, N. (1986). Semiconductor Lasers, (1 ${ }^{\text {st }}$ Edition), Van Nostrand Reinhold, ISBN: 0-442-20995-9, N.Y.

Boavida, J., Morgado, J. \& Fernandes, C. HR-AR, coated DFB lasers with high-yield and enhanced above-threshold performance, Optics and Laser Technology, 43, 2011, pp. 729735.

Bornholdt C., Troppenz, U, Kreissl, J., Rehbein, W., Sartorius, B., Schell, M. \& Woods, I. 40Gbit/s directly modulated passive feedback DFB laser for transmission over 320 $\mathrm{km}$ single mode fibre, Proc. 34th European Conference on Optical Communication (ECOC'08), Vol. 2, Brussels, Belgium, 2008.

Coldren, L. \& Corzine, S. (1995). Diode Lasers and Photonic Integrated Circuits, (1st Edition), John Wiley \& Sons, Inc., ISBN: 0-471-11875-3), USA.

Davis, M. \& O' Dowd, R. A Transfer Matrix-Based Analysis of Multielectrode DFB Lasers, IEEE Photonics Technology Letters, Vol. 3, No. 7, 1991, pp. 603-605.

Davis, M. \& O' Dowd, R. A New Large-Signal Dynamic Model for Multielectrode DFB Lasers Based on the Transfer Matrix Method, IEEE Photonics Technology Letters, Vol. 4, No. 8, 1992, pp. 838-840.

Fernandes, C, Morgado, J. \& Boavida, J. Optimisation of an asymmetric three phase-shift distributed feedback semiconductor laser, EPJ AP Applied Physics, 46, 2009, p.30701.

Fessant, T. Threshold and Above-Threshold Analysis of Corrugation-Pitch Modulated DFB Lasers with Inhomogeneous Coupling Coefficient, , IEE. Proc. Optoelectron, Pt J, 144(6), 1997, pp. 365-376.

Fessant, T. Influence of a nonuniform coupling coefficient on the static and large signal dynamic behavior of Bragg-detuned DFB lasers, J. Ligthwave Techn. Vol.16, no.3, 1998, pp. 419-427.

Ghafouri-Shiraz, H. (2003). Distributed Feedback Laser Diodes and Optical Tunable Filters, (1st Edition), J. Wiley \& Sons, ISBN: 0-470-85618-1, Chichester.

Jia, X., Zhong, D., Wang, F., Chen, H. Detailed modulation response analysis on enhanced single-mode QWS-DFB lasers with distributed coupling coefficient, Optics Communications, 277, 2007, pp. 166-173.

Kapon, E., Hardy, A. \& Katzir, A. The effect of complex coupling coefficients on distributed feedback lasers, IEEE J. Quantum Electron., 18, 1982, pp.66-71.

Kim, Y. \& Jeong, J. Analysis of Large-Signal Dynamic Characteristics of 10-Gb/s Tunable Distributed Bragg Reflector Lasers Integrated With Electroabsorption Modulator and Semiconductor Optical Amplifier Based on the Time-Depenedent Transfer Matrix Method, IEEE Journal of Quantum Electronics, Vol. 39, No. 10, 2003, pp. 13141320.

Kogelnik, H. \& Shank, C. Coupled-wave theory of distributed feedback lasers, J. Appl. Phys. 43(5), 1972, pp. 2327-2335.

Lee, H., Yoon, H., Kim, Y. \& Jeong, J. Theoretical Study of Frequency Chirping and Extinction Ratio of Wavelength-Converted Optical Signals by XGM and XPM Using SOA's, IEEE Journal of Quantum Electronics, Vol. 35, No. 8, 1999, pp. 1213-1219.

Lowery, A. Integrated mode-locked laser design with a distributed-Bragg reflector, IEEProceedings, Pt. J, 138(1), 1991, pp.39-46.

Morthier, G. \& Vankwikelberge, P. (1997). Handbook of Distributed Feedback Laser Diodes, (1st Edition) Artech House, ISBN: 0-89006-607-8, Norwood. 
Sato, K., Kuwahara, S. \& Miyamoto, Y. Chirp characteristics of 40-Gb/s directly modulated distributed-feedback laser diodes, IEEE/OSA J. Lightwave Technology, Vol. 23, No.11, 2005, pp. 3790-3796.

Tan, P., Ghafouri-Shiraz, H. \& Lo, B. Theoretical analysis of multiple-phase-shift controlled DFB wavelength tunable optical filters, Microwave Opt. Technol. Letters, 8(2), 1995, pp.72-75.

Tang, J., Lane, P. \& Shore, K. High speed transmission of adaptively modulated optical OFDM signals over multimode fibres using directly modulated DFBs, IEEE/OSA J. Lightwave Technology, Vol. 24, No. 1, 2006, pp. 429-441.

Utake, A., Otsubo, K., Matsuda, M., Okumura, S., Ekawa, M. \& Yamamoto, T. 40 Gbps direct modulation of $1.55-\mu \mathrm{m}$ AlGaInAs semi-insulating buried-heterostructure distributed reflector lasers up to $85^{\circ} \mathrm{C}$, Proc. 2nd Annual Meeting of the IEEE Photonics Society, Vol. 1, Antalya, Turkey, 2009.

Wedding, B., Pöhlmann, W., Gross, H. \& Thalau, O. 43 Gbit/s transmission over 210 km SMF with a directly modulated laser diode, Proc. 29th European Conference Optical Communication (ECOC'03), Rimini, Italy, 2003, 2003.

Wedding, B. \& Pöhlmann, W. 43Gbit/s transmission over $40.5 \mathrm{~km}$ SMF without optical amplifier using a directly modulated laser diode, Proc. 30th European Conf. Optical Communication (ECOC'04), Stockholm, Sweden, 2004.

Yu,S. (2003). Analysis and Design of Vertical Cavity Surface Emitting Lasers, (1'st Edition), Wiley, series in Lasers and applications, ISBN: 0-471-39124-7, New Jersey. 


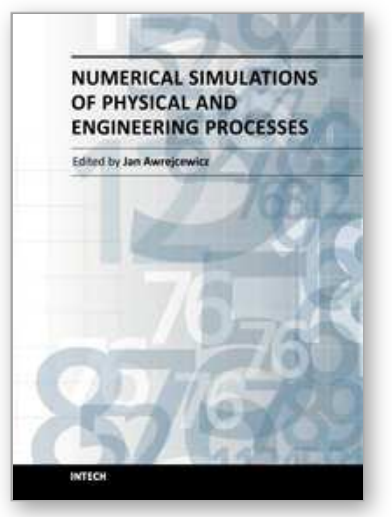

\author{
Numerical Simulations of Physical and Engineering Processes \\ Edited by Prof. Jan Awrejcewicz
}

ISBN 978-953-307-620-1

Hard cover, 594 pages

Publisher InTech

Published online 26, September, 2011

Published in print edition September, 2011

Numerical Simulations of Physical and Engineering Process is an edited book divided into two parts. Part I devoted to Physical Processes contains 14 chapters, whereas Part II titled Engineering Processes has 13 contributions. The book handles the recent research devoted to numerical simulations of physical and engineering systems. It can be treated as a bridge linking various numerical approaches of two closely interrelated branches of science, i.e. physics and engineering. Since the numerical simulations play a key role in both theoretical and application oriented research, professional reference books are highly needed by pure research scientists, applied mathematicians, engineers as well post-graduate students. In other words, it is expected that the book will serve as an effective tool in training the mentioned groups of researchers and beyond.

\title{
How to reference
}

In order to correctly reference this scholarly work, feel free to copy and paste the following:

C. A. F. Fernandes and José A. P. Morgado (2011). The Static and Dynamic Transfer-Matrix Methods in the Analysis of Distributed-Feedback Lasers, Numerical Simulations of Physical and Engineering Processes, Prof. Jan Awrejcewicz (Ed.), ISBN: 978-953-307-620-1, InTech, Available from:

http://www.intechopen.com/books/numerical-simulations-of-physical-and-engineering-processes/the-staticand-dynamic-transfer-matrix-methods-in-the-analysis-of-distributed-feedback-lasers

\section{INTECH}

open science | open minds

\author{
InTech Europe \\ University Campus STeP Ri \\ Slavka Krautzeka 83/A \\ 51000 Rijeka, Croatia \\ Phone: +385 (51) 770447 \\ Fax: +385 (51) 686166 \\ www.intechopen.com
}

\author{
InTech China \\ Unit 405, Office Block, Hotel Equatorial Shanghai \\ No.65, Yan An Road (West), Shanghai, 200040, China \\ 中国上海市延安西路65号上海国际贵都大饭店办公楼 405 单元 \\ Phone: +86-21-62489820 \\ Fax: $+86-21-62489821$
}


(C) 2011 The Author(s). Licensee IntechOpen. This chapter is distributed under the terms of the Creative Commons Attribution-NonCommercialShareAlike-3.0 License, which permits use, distribution and reproduction for non-commercial purposes, provided the original is properly cited and derivative works building on this content are distributed under the same license. 